\title{
Rectification in Nonequilibrium Parity Violating Metamaterials
}

\author{
Zhenghan Liao $\circledast^{1}{ }^{1}$ William T. M. Irvine, ${ }^{2,3,4}$ and Suriyanarayanan Vaikuntanathan ${ }^{1,3, *}$ \\ ${ }^{1}$ Department of Chemistry, University of Chicago, Chicago, Illinois 60637, USA \\ ${ }^{2}$ Department of Physics, University of Chicago, Chicago, Illinois 60637, USA \\ ${ }^{3}$ James Franck Institute, University of Chicago, Chicago, Illinois 60637, USA \\ ${ }^{4}$ Enrico Fermi Institute, University of Chicago, Chicago, Illinois 60637, USA
}

(Received 5 September 2019; revised manuscript received 5 March 2020; accepted 26 March 2020; published 15 May 2020)

\begin{abstract}
Understanding mechanisms for rectifying stochastic fluctuations has been a long-standing problem in nonequilibrium statistical mechanics. Here, we explore an opportunity provided by nonequilibrium parityviolating metamaterials to uncover new mechanisms for rectification of energy and motion. Using a parityviolating gyroscopic metamaterial that is allowed to interact with a bath of active particles as a model system, we develop an analytic diagrammatic theory that compactly elucidates how the rectification results from an interplay between gyroscopic forces, nonequilibrium activity, and network structure. Our active metamaterial model can generate energy flows through an object in the absence of any temperature gradient. The nonreciprocal microscopic fluctuations responsible for generating the energy flows can further be used to power locomotion in, or exert forces on, a viscous fluid. Taken together, our analytical and numerical results elucidate how the geometry and interparticle interactions of the parity-violating material can couple with the nonequilibrium fluctuations of an active bath and enable rectification of energy and motion.
\end{abstract}

DOI: 10.1103/PhysRevX.10.021036

Subject Areas: Metamaterials, Statistical Physics

\section{INTRODUCTION}

Identifying mechanisms that rectify stochastic fluctuations is a long-standing problem in nonequilibrium statistical mechanics, with many biological, chemical, and physical applications [1-5]. For instance, the Feynman's ratchet-and-pawl model [6] and its associated extensions have provided a framework to explain how biological molecular motors can convert the energy derived from the hydrolysis of energy-rich molecules into mechanical work [2-5,7-10]. Substantial advances have been made in understanding the rectification of motion from unbiased nonequilibrium fluctuations along with spatial or temporal symmetry breaking $[4,11,12]$. In this article, we focus on directed energy conduction in the absence of temperature biases, which, despite pioneering works [13-25], remains less well explored. Compared with directed motion whose basic mechanism can be understood in the single-body basis [4], conductive transport of energy is, by definition, a many-body effect.

*svaikunt@uchicago.edu

Published by the American Physical Society under the terms of the Creative Commons Attribution 4.0 International license. Further distribution of this work must maintain attribution to the author(s) and the published article's title, journal citation, and DOI.
We exploit an opportunity provided by the combination of parity-violating metamaterials and nonequilibrium fluctuations to uncover new many-body principles for energy rectification. In particular, we consider a class of springmass networks that are subject to Lorentz forces, immersed in a bath of active particles [26-28]. Using numerical calculations and simulations, we reveal the presence of net energy fluxes at the nonequilibrium steady state, whose origin we study analytically. Our central analytical results, compactly represented as a diagrammatic expansion for the energy fluxes, show how nonequilibrium metamaterials with parityviolating dynamics are able to rectify energy and motion when allowed to interact with a bath of active particles.

The diagrammatic approach reveals a hierarchical picture of the energy transport, starting from lowest-order diagrams that only depend on local structures of the network to higher-order diagrams that involve more and more nonlocal structures. Importantly, the leading-order diagrams describe the many-body effect in a compact analytic form, Eq. (12), that elucidates how a simple interplay between the geometry, activity, and parity violation enable rectification. The diagrammatic expansion, even at the lowest order, provides concrete design principles for controlling the rectification of energy by engineering the geometry of our metamaterials.

We note in passing that undamped variants of our active parity-violating metamaterials were recently exploited to generate chiral (topological) eigenmodes [29-32]. However, 
our energy flux is not related to the topological properties (if any) of the undamped mechanical systems due to the strong roles played by friction (damping) and broad excitation of the modes by nonequilibrium fluctuations.

Unlike conventional energy flows, our energy flow does not require a temperature gradient. This flux can be routed through an otherwise isolated passive object, with the active network acting as a current source. Finally, we show that the microscopic mechanisms responsible for this energy flow can, in principle, allow the passive object to swim in or pump a viscous fluid. Taken together, our results establish a new mechanism for rectification of energy and motion in nonequilibrium parity-violating metamaterials. Unlike existing prescriptions, our mechanism exploits inherent asymmetries in the nonequilibrium fluctuations of the active bath, parity-violating dynamics, and network geometry to achieve rectification.

The remainder of this paper is organized as follows. In Sec. II, we introduce our model parity-violating metamaterial and provide a microscopic definition of the energy flux. In Secs. III-V, we analytically identify the ingredients for rectification of energy fluxes and construct a diagrammatic approach that reveals a relationship between energy flux and network geometries. Finally in Secs. VI and VII, we show that when the particles transmitting the energy flux are allowed to interact with a viscous low Reynolds number fluid, the nonreciprocal motions responsible for the energy flux can be utilized to pump the viscous fluid.

\section{MODEL SYSTEMS AND ENERGY FLUX}

Our model is a tethered spring-mass network in which each particle is additionally subject to a Lorentz-like force and stochastic forcing from an active bath [Fig. 1(a)]. The linearized equation of motion is

$$
m \dot{v}_{i}=-k_{g} z_{i}+\sum_{j} F_{j i}-\hat{B} A_{1} v_{i}-\gamma v_{i}+\eta_{i} .
$$

Here, $z_{i} \equiv\left(x_{i} y_{i}\right)^{T}$ is a column vector that denotes the displacement of particle $i$ from its mechanical equilibrium position, and similarly, $v_{i}$ and $\eta_{i}$ describe the $2 \mathrm{D}$ velocity of and noise on each particle. Note that $-k_{g} z_{i}$ is the on-site tethering force used to maintain the shape of the network, and $F_{j i}=k\left(e_{i j}^{T} z_{i}+e_{j i}^{T} z_{j}\right)\left(-e_{i j}\right)$ is the linearized spring force from $j$ to $i$, where $e_{i j}$ is the unit vector that points from the equilibrium position of $i$ to that of $j$. In addition, $-\hat{B} A_{1} v_{i}=-\hat{B}\left(v_{i, y}-v_{i, x}\right)^{T}$ is the Lorentz-like force, where $\hat{B}=e B$ is the product of the electric charge $e$ and the magnetic field $B$, and the matrix

$$
A_{1} \equiv\left(\begin{array}{cc}
0 & 1 \\
-1 & 0
\end{array}\right)
$$

The last two terms describe the forcing from the active bath, which consists of the friction $-\gamma v_{i}$ and an Ornstein-
Uhlenbeck (OU) colored noise $\eta_{i}$ [33]. The colored noise has finite correlation time $\tau$, with statistics

$$
\left\langle\eta_{i}(t) \eta_{j}^{T}\left(t^{\prime}\right)\right\rangle=I \delta_{i j} \frac{\gamma T_{a}}{\tau} e^{-\left(\left|t-t^{\prime}\right| / \tau\right)},
$$

where, for fixed $\tau$, the parameter $T_{a}$ controls the variance of the colored noise, and $I$ is the identity matrix with appropriate dimensions. The time evolution of the OU noise can be described according to the following equation [33],

$$
\tau \dot{\eta}_{i}=-\eta_{i}+\sqrt{2 \gamma T_{a}} \xi_{i},
$$

where $\xi_{i}$ is a delta-function-correlated white noise with unit variance. The friction $-\gamma v_{i}$ and $\mathrm{OU}$ noise $\eta_{i}$ break the fluctuation-dissipation relation, thus driving the system out of equilibrium. The active bath reduces to a thermal equilibrium bath in the $\tau \rightarrow 0$ limit. The construction of our model system is motivated by recently constructed topological gyroscopic metamaterials [29], although the presence of friction and active noise make the dynamics of our system significantly different from those of undamped gyroscopic metamaterials. In the linearized regime, the dynamics of gyroscopes is mathematically equivalent to that of particles subject to a Lorentz force and an on-site potential [31].

Since the particles in our model are tethered by on-site potentials, rectification of fluctuations, if any, does not result in any particle flows. Rather, rectified fluctuations can affect the transport of energy. To study such phenomena, the observable we mainly focus on is the timeaveraged energy flux between particles at the steady state. For a system with pairwise interactions and on-site potentials, the energy flux $\left\langle J_{i j}\right\rangle$ from particle $i$ to $j$ reads

$$
\left\langle J_{i j}\right\rangle=\left\langle\frac{1}{2}\left(v_{j}^{T} F_{i j}-v_{i}^{T} F_{j i}\right)\right\rangle=\left\langle v_{j}^{T} F_{i j}\right\rangle .
$$

To arrive at this formula, we first define the energy of a particle as the sum of its kinetic energy, on-site potential energy, and one-half of the bond energies [34]. Then, we write down the energy balance relations using stochastic energetics [35]. Finally, we identify the energy exchanged due to particle-particle interactions as the energy flux $\left\langle J_{i j}\right\rangle$ (derived in detail in the Appendix A). We note that the energy flux can simply be interpreted as the rate at which work is done on particle $j$ by particle $i$. Since this microscopic work is due to particles' stochastic motions, rather than due to an external control, the energy flux can also be interpreted as a heat flux [34,35]. The averaged energy fluxes, Eq. (4), are identically equal to zero for a system at equilibrium with Boltzmann distribution.

Starting from the linear equations (1) and (3), the energy fluxes can be solved numerically using methods introduced in Ref. [36,37] (Appendix A). A collection of numerical results are shown in Figs. 1(b) -1 (d). We see that nonzero 
(a)

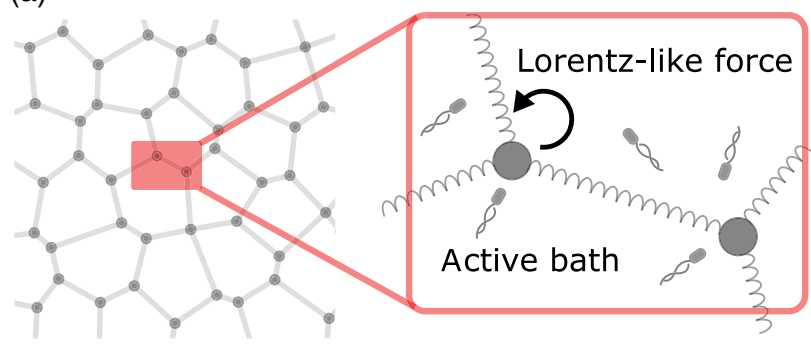

(b)

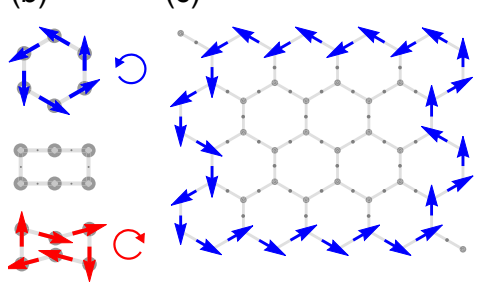

(d)

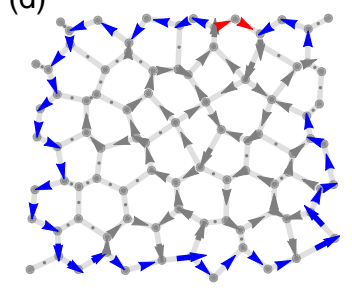

FIG. 1. The model and the energy flux in example networks. (a) Schematic of the model, a spring-mass network subject to a Lorentz-like force and immersed in an active bath. (b)-(d) Averaged energy flux from numerical calculations for example networks. The flux direction and pattern can be controlled by the network geometry. In these figures, gray lines and dots represent the mechanical equilibrium structure of the network, and the arrows represent the flux between particles, whose length is proportional to the flux magnitude. An arrow is colored blue if it is counterclockwise (CCW), red if clockwise (CW), and gray for fluxes not on the boundary. The numerical calculations were performed with all parameters set to 1 .

energy rectification or energy fluxes can be generated in our chiral active system, and the flux direction or pattern changes with the network geometry. Using a linear response theory, we now develop analytical expressions for the energy flux that reveal how a combination of chirality, nonequilibrium activity, and network geometry is responsible for generating energy fluxes.

\section{LINEAR RESPONSE THEORY FOR ENERGY FLUX}

We begin by writing the equations of motion, Eq. (1), in frequency space,

$$
\begin{gathered}
\tilde{z}(\omega)=G^{+}(\omega) \tilde{\eta}(\omega), \\
G^{+}(\omega) \equiv\left[K+i \omega(\gamma I+\hat{B} A)-m \omega^{2} I\right]^{-1} .
\end{gathered}
$$

Here, we have represented the displacement of all $N$ particles using a $2 N$-dimensional column vector $z=$ $\sum_{i}|i\rangle \otimes z_{i}$, with $|i\rangle$ denoting the 2D subspace corresponding to particle $i$. Note that $\tilde{z}(\omega)$ and $\tilde{\eta}(\omega)$ denote the Fourier transform of $z$ and the OU noise $\eta$, respectively; $G^{+}$is the response matrix, in which matrix $K$ encodes all on-site and spring forces $F$ according to $F=-K z$; and $A$ is an

antisymmetric matrix $A=\sum_{i}|i\rangle\langle i| \otimes A_{1}$. Equation (5) describes how the displacement responds to the noise.

Following the procedure in Ref. [38], the flux defined in Eq. (4) can be expressed using $G^{+}$as a spectral integral (Appendix B),

$$
\begin{gathered}
\langle J\rangle=\int_{-\infty}^{\infty} d \omega h(\omega) J^{F T}(\omega), \\
J^{F T}(\omega) \equiv-\frac{T_{a} k}{2 \pi} \operatorname{Re} \operatorname{tr} G^{+}(\omega) A^{\text {as }}, \\
h(\omega)=\frac{1}{1+\omega^{2} \tau^{2}},
\end{gathered}
$$

where $A^{\text {as }}$ is an antisymmetric matrix $A^{\text {as }}=-|i\rangle\langle j| \otimes$ $e_{i j} e_{j i}^{T}+|j\rangle\langle i| \otimes e_{j i} e_{i j}^{T}$. The response function $G^{+}(\omega)$ has no pole in the lower-half of the complex plane, but the colored noise introduces one pole at $\omega=-i / \tau$. Using the residue theorem, we get a compact expression for the energy flux (Appendix B),

$$
\frac{\langle J\rangle}{T_{a} / \tau}=-\frac{k}{2} \operatorname{tr} G^{+}\left(-\frac{i}{\tau}\right) A^{\text {as }} .
$$

Higher-order moments of the (integrated) energy flux can also be calculated, which we present in Appendix B 3 .

Equations (7) and (10) will serve as our starting point to understand the energy flux. While they contain all the information required to compute energy fluxes, they have limited utility as design principles. Indeed, as written down, they require that the flux be recomputed de novo for each new network geometry and nonequilibrium bath activity. In the next two sections, we show that it is possible to expand Eqs. (7) and (10) in forms that reveal design principles for controlling energy fluxes.

Interestingly, one important property that can be directly obtained from a similar linear response analysis is that the energy fluxes satisfy Kirchoff's law, $\sum_{i}\left\langle J_{i j}\right\rangle=0$. Kirchoff's law shows that, on average, there is no net energy exchange between particles and the active bath. To derive Kirchoff's law, we calculate the average heat exchange between particle $i$ and the active bath $\left\langle v_{i}^{T} \eta_{i}-\gamma v_{i}^{T} v_{i}\right\rangle$, and following the procedures in Ref. [38], this heat exchange can be shown to be zero (Appendix C). Kirchoff's law places a strong constraint on possible energy flux patterns between particles, and some corollaries immediately follow-such as networks with no cycles cannot have nonzero flux, and fluxes of all bonds in a polygon network [as in Fig. 1(b)] are equal.

Finally, we note that Eq. (7) is distinct from a Harada-Sasalike relation [39] that connects the entropy production rate to the extent of violation of the fluctuation dissipation theorem. Indeed, when the $B$ field is turned off, the energy flux vanishes while the entropy production rate does not, on account of the nonequilibrium active bath. For completeness, 
in Appendix D, we present a Harada-Sasa relation for our system.

\section{INGREDIENTS FOR ENERGY RECTIFICATION AND THEIR ROLES}

Compared with an ordinary thermal spring-mass network, which supports no energy fluxes in its equilibrium state, our active gyroscopic network contains two extra components- the Lorentz force and the correlation in the noise. We now show that these two components provide two necessary ingredients required to ensure energy rectification in our model.

\section{A. Lorentz force and nonequilibrium activity are necessary for the generation of an energy flux}

We begin with Eq. (7), which represents the averaged flux in terms of functions $J^{F T}(\omega), h(\omega)$. In particular, we note that the function $J^{F T}(\omega)$ is proportional to the energy flux at Fourier frequency $\omega$ in an isolated damped variant of our network, while the function $h(\omega)$ is proportional to the noise spectrum, $\left\langle\tilde{\eta}(\omega) \tilde{\eta}(\omega)^{*, T}\right\rangle=2 \gamma T_{a} \operatorname{Ih}(\omega) / t$.

To generate a nonzero flux, or equivalently make the integral nonzero in Eq. (7), we have two requirements (Fig. 2). First, $J^{F T}(\omega)$ should not be zero everywhere. In the absence of a magnetic field, $B=0$, it can be easily shown that $J^{F T}(\omega)=0$. Indeed, the response function $G^{+}$ is symmetric or reciprocal when $B$ is absent, and since

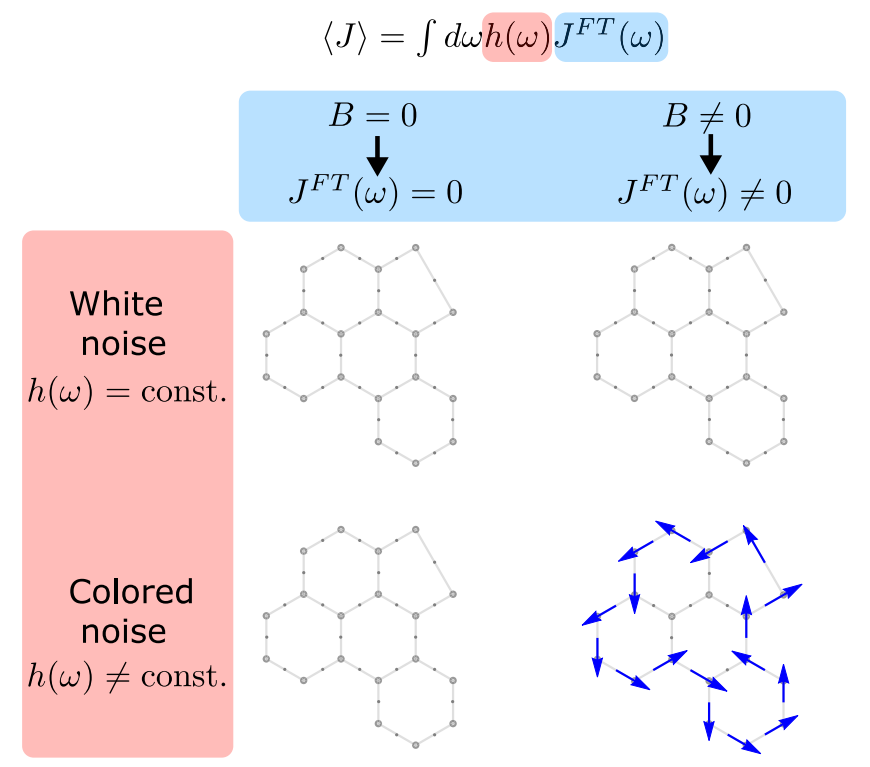

FIG. 2. Necessary ingredients for generating nonzero energy fluxes. Both the Lorentz-like force and colored noise are needed to generate nonzero fluxes. The role of the Lorentz-like force is to provide chiral Fourier modes $\left(J^{F T}(\omega) \neq 0\right)$. If $B=0, J^{F T}(\omega)$ is zero everywhere. The role of colored noise is to provide weighted excitation $h(\omega) \neq$ const, which makes a nonvanishing averaged flux $\langle J\rangle$ possible. The numerical calculations were performed with $m, k_{g}, k, \gamma, T_{a}=1$.
$A^{\text {as }}$ is antisymmetric, the trace $\operatorname{tr} G^{+}(\omega) A^{\text {as }}=0$ at all values of $\omega$. We note that the chirality of network geometry does not affect the reciprocity of the response matrix; i.e., chiral networks without a $B$ field also lead to $J^{F T}(\omega)=0$. Nonzero $B$ breaks the reciprocity of $G^{+}$and can thus generate a nonzero $J^{F T}(\omega)$.

Nonzero $J^{F T}(\omega)$ alone does not, however, ensure a nonzero averaged energy flux; in fact, we must further require that $h(\omega)$ is not constant. Indeed, noise characterized by a constant $h(\omega)$ function corresponds to fluctuation dissipation preserving white noise. In such cases, our model system would be in equilibrium even in the presence of a magnetic field, according to the Bohr-van Leeuwen theorem [40]. Mathematically, if $h(\omega)$ is constant, the integrand in Eq. (7) has no poles in the lower-half $\omega$ plane because $G^{+}(\omega)$ is a linear response function, which satisfies causality. The absence of poles ensures that the flux integral vanishes. A nonconstant $h(\omega)$ function corresponding to a colored noise source can support a nonzero average energy flux through its introduction of poles. In this paper, we focus on a specific choice of the colored noise, namely, the OU colored noise. The spectrum of OU noise is a Lorentzian, $h(\omega)=1 /\left(1+\omega^{2} \tau^{2}\right)$, which excites lower frequency modes with larger weights.

In summary, we see that a $B$ field and colored noise are two necessary ingredients to generate energy fluxes in our model chiral system. The role of the $B$ field is to break the reciprocity of response and generate Fourier modes such that $J^{F T}(\omega) \neq 0$. The role of the colored noise is to excite these modes in a weighted manner.

\section{B. Comments on the relation to undamped isolated gyroscopic metamaterials}

The energy flux in our damped metamaterials driven by nonequilibrium fluctuations cannot simply be related to the eigenmodes of the isolated undamped variant [29,32]. This idea is manifest when comparing energy transport in a honeycomb lattice with that in a triangular lattice. The undamped, topologically nontrivial, honeycomb lattice of gyroscopes supports chiral edge transport, whereas the topologically trivial triangular lattice of gyroscopes does not [29]. By contrast, in the presence of damping and nonequilibrium forcing, both honeycomb [Fig. 1(c)] and triangular lattices generate chiral energy fluxes localized on the boundary. This difference demonstrates that the existence of topology in the band structure of the isolated undamped variant of the network is not a controlling factor in establishing our energy fluxes.

The chiral eigenmodes in the undamped gyroscopic metamaterials, however, can provide an intuition for how $J^{F T}(\omega)$ can be chiral in the small damping regime. Specifically, the weakly damped dynamics resonate near the eigenfrequencies of the undamped metamaterials and hence exhibit Fourier modes that resemble the eigenmodes of the undamped system. In the regime of strong damping 
or generic damping, an emergent connection can be built between energy fluxes in the active system and all the eigenmodes of a reference isolated system where the onsite harmonic potential is modified (Appendix E). While this emergent connection reveals contributions from each modified eigenmode, it does not result in an intuitive framework that reveals how the interplay between geometry, Lorentz forces, and nonequilibrium activity controls energy rectification. In the next section, we develop a diagrammatic technique that achieves this result.

\section{A DIAGRAMMATIC APPROACH TO COMPUTE THE ENERGY FLUX}

In this section, we develop a diagrammatic technique that provides a simple intuitive method to compute energy fluxes and elucidates the relationship between flux and network geometry. The diagrammatic technique is constructed by expanding the expressions for the energy flux, Eq. (10), in the small- $k$ regime, and it shows how the energy flux across a bond can be expressed as a sum over paths traversed along the network [Eq. (11)]. Our perturbation theory assigns a geometry-dependent prefactor for each path, thus elucidating the role played by network geometry in ensuring rectified energy fluxes [Eq. (12)]. Together, the central results of this section, summarized in Eqs. (11) and (12), provide compact expressions that elucidate how geometry, the $B$ field, and correlation time $\tau$ of the colored noise can combine to generate energy flows in networks with arbitrarily complex geometry and topology. The lowest-order expansion in Eq. (12) demonstrates, for instance, how the parameter $\alpha$ that compactly captures the combined effect of activity, the $B$ field, couples with the geometry of the network to power the flow of energy. The diagrammatic expansion, even at the lowest order, hence results in a concrete design principle for controlling the rectification of the energy by our metamaterial.

\section{A. Diagrammatic expansion, diagram rules, and properties}

The starting point of the diagrammatic approach is the flux formula, Eq. (10). First, we expand matrix $G^{+}(-i / \tau)$ to different orders in the spring constant $k$; then, the blockmatrix structure in each term enables further decomposition into paths, and the structure of matrix $A^{\text {as }}$ closes these paths into cycles (Appendix F1). As a result, the flux is decomposed into a series of terms,

$$
\frac{\langle J\rangle}{T_{a} / \tau}=\sum_{l} J_{l}^{\text {path }}
$$

where each term can be pictorially represented as a closed path or a diagram as described in Fig. 3(a). The full expression for $J_{l}^{\text {path }}$ is lengthy and is detailed in Appendix F 1. However, when restricted to leading order

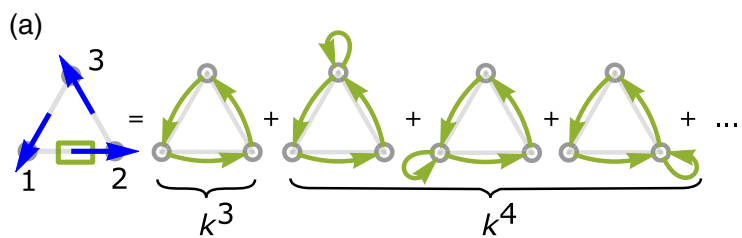

(b)

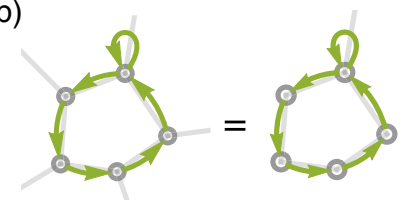

(c)

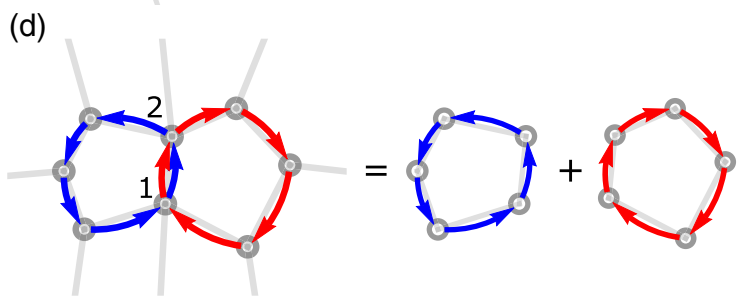

FIG. 3. Illustrations of our diagrammatic technique. (a) Flux from site 1 to site 2 can be calculated by summing over diagrams. Each diagram is a closed path, which pictorially represents one term in the small- $k$ expansion. Paths are depicted using green arrows. The magnitude of the path of length $n$ is on the order of $k^{n}$. (b) A useful property of the diagram is that if there is no loop on a node, then the diagram can be simplified by removing all other branches on the node. (c) Schematic of a polygon path and its outer angles $\theta_{1}, \theta_{2}, \ldots, \theta_{n}$. The value of this diagram is written in Eq. (12). (d) The lowest-order flux from 1 to 2 in complex networks is the sum over the shortest cycles that contain $1 \rightarrow 2$, which in this schematic are two pentagon diagrams $\left(k^{5}\right)$. If, for instance, one of the pentagons is replaced by a triangle, then the triangle diagram $\left(k^{3}\right)$ dominates the flux from 1 to 2 .

polygon diagrams, its form simplifies significantly as detailed in the next subsection.

The rule for constructing diagrams is as follows. For the flux from site $i$ to $j$, the valid paths are $l: i \rightarrow j \rightarrow$ $l_{3} \rightarrow l_{4} \rightarrow \ldots \rightarrow l_{n} \rightarrow i$, where the consecutive sites have to be either bonded or identical. The latter case is depicted as a loop in the diagram. Paths that contain equal numbers of $i \rightarrow j$ and $j \rightarrow i$ do not contribute (e.g., path $i \rightarrow j \rightarrow i$ ) because either the path itself vanishes or it cancels with another valid path. As a consequence, paths have to at least traverse a polygon in the network.

Paths with length $n$ are on the order of $k^{n}$. In the small- $k$ regime, major contributions to fluxes come from shorter paths. A useful property of the diagrams is that if there is no loop on a node, then the diagram can be simplified by removing all other branches on the node [Fig. 3(b), Appendix F 2]. The diagrammatic expansion hence reveals a hierarchical picture of the energy transport, starting from lowest-order diagrams that only depend on local structures of the network (nonlocal nodes are removed in simplified diagrams), to higher-order diagrams that involve more and more nonlocal structures. 


\section{B. Diagrammatic approach reveals connections between rectification and geometry, activity, parity breaking}

To illustrate how the diagrammatic approach clarifies the connection between geometry, activity, parity breaking, and rectification, we consider the generic, dominant, lowestorder diagrams, which are polygonal cycles with no loops. The expression for the flux of these polygon paths reduces to a compact form (Appendix F 3)

$$
J_{\text {polygon }}^{\text {path }}=\frac{1}{2}\left(\frac{k}{k_{0}}\right)^{n}\left(\prod_{i} \cos \left(\theta_{i}-\alpha\right)-\prod_{i} \cos \left(\theta_{i}+\alpha\right)\right),
$$

where $n$ is the number of nodes and $\theta_{i}$ 's are outer angles of the polygon [Fig. 3(c)]. Here, $\alpha$ is defined as

$$
\alpha \equiv \arcsin \frac{\hat{B} / \tau}{k_{0}},
$$

where $k_{0} \equiv \sqrt{\left(k_{g}+\gamma / \tau+m / \tau^{2}\right)^{2}+(\hat{B} / \tau)^{2}}$ sets a characteristic scale for the interaction $k$. This single auxiliary angle $\alpha$ encodes information from all the parameters, $m, k_{g}$, $k, \hat{B}, \gamma, \tau$ (except for $T_{a}$, which simply sets the energy scale), in a condensed manner.

The expression (12) illustrates how the flux results from a combination of the geometry of the network, as characterized by angles $\theta_{i}$, together with the condensed parameter $\alpha$ that encodes the nonreciprocity due to the $B$ field and the violation of fluctuation dissipation due to the colored noise. It is surprising to see that the many-body effect can be expressed in such a compact manner. As an example, applying Eq. (12) to an arbitrary triangle results in $J_{\text {polygon }}^{\text {path }} \propto k^{3} \sin \alpha \sin \theta_{1} \sin \theta_{2} \sin \theta_{3}$, which is always positive or CCW if $\alpha>0$. As a consistency check, the flux from Eq. (12) should vanish in the limit of zero $\tau$, to be consistent with the expected equilibrium behavior. If $m$ is finite, as $\tau$ tends to zero, the parameter $\alpha \rightarrow 0$, and consequently, $\langle J\rangle \rightarrow 0$. If we take $m \rightarrow 0$ first, as $\tau$ reduces to zero, $\alpha$ remains finite. However, we still get $\langle J\rangle \rightarrow 0$ due to its vanishing prefactor, $\langle J\rangle \propto 1 / \tau k_{0}^{n} \sim \tau^{n-1}$.

For complex networks, lowest-order fluxes can be viewed as a result of combining the flux of their constituent polygons, as illustrated in Fig. 3(d). From the property demonstrated in Fig. 3(b) or from Eq. (12), polygon diagrams are not affected by any side chains on its nodes; thus, $J_{\text {polygon }}^{\text {path }}$ for a polygon in a complex network is equal to $J_{\text {polygon }}^{\text {path }}$ for the polygon by itself.

The diagrammatic approach, when applied to complex networks, can explain the various emergent flux patterns seen in numerical calculations. In the example of ordered honeycomb networks [Fig. 1(c)], fluxes are localized at the boundary of the network, which can be explained as follows. The lowest-order diagram for a bond at the boundary is one hexagon path, which contributes a flux on the order of $k^{6}$. In contrast, the lowest-order diagrams for a bond in the bulk are two paths, each traversing the hexagon on one side of the bond. The contributions from these two diagrams cancel each other; thus, the flux on the order of $k^{6}$ is zero. However, contributions from higherorder diagrams persist, and they show that the flux exhibits an exponential decay into the bulk. The decay length can be expressed analytically in the small- $k$ limit using the diagrammatic approach (Appendix F4). We clarify that such localization is not a general feature for all crystalline networks. As a counterexample, the kagome lattice also supports fluxes in its bulk in triangle units.

In the example of disordered networks [Fig. 1(d)], fluxes are generated throughout the network because each unit polygon is different; hence, there is no cancellation of diagrams. The noncancellation effect is stronger when neighboring polygon units have a different number of sides, which would contribute to diagrams on different orders in $k$.

If one is able to sum over all the diagrams to all orders, the exact result can be obtained. Such a result holds beyond the small- $k$ regime due to analytic continuation. One example is the 1D network, where we can show that all diagrams vanish in Appendix F5. Another example is shown in Sec. VII A.

\section{Generalization of the diagrammatic approach to higher-dimensional networks, generic noise spectrum, and heterogeneous parameters}

We have seen how the diagrammatic approach reveals a hierarchical picture of the energy transport [Eq. (11)] and elucidates how the Lorentz force and activity, operating through the condensed parameter $\alpha$, interact with the geometry to enable rectification [Eq. (12)]. Together, these results make it possible to control energy fluxes in arbitrarily complex networks.

We now show that the diagrammatic approach can enable control in a wider variety of systems. Our results can immediately be generalized to networks on $2 \mathrm{D}$ curved surfaces or to 3D networks. For 2D curved surfaces, the same diagrammatic expressions, Eqs. (11) and (12), can be readily applied. Curvature simply modifies the allowed geometry as characterized by the outer angles $\left\{\theta_{i}\right\}$ and the number of sides $n$. For 3D networks, the same diagrammatic approach applies, but the expression would be modified due to the additional spatial dimension. Examples of networks on 2D curved surface or in 3D space are shown in Appendix G 1 .

The OU noise spectrum can be generalized to $\left\langle\tilde{\eta}(\omega) \tilde{\eta}(\omega)^{*, T}\right\rangle=2 \gamma T_{a} I h(\omega) / t$ with a generic $h(\omega)$. The diagrammatic approach still applies, except now the real matrix $G^{+}(\omega=-i / \tau)$ is replaced by the complex matrix $G^{+}(\omega)$. As a result, the path rules remain unchanged, but the mathematical expression corresponding to each diagram is modified. In Appendix G 2, we show a 
diagrammatic expansion of $J^{F T}(\omega)$. Consequently, the flux can be expressed as a weighted integral of $J^{F T}(\omega)$ over $\omega$ or $J^{F T}(\omega)$ evaluated at the poles.

The diagrammatic approach can be extended to calculate energy fluxes in heterogeneous networks where all parameters except for those related to the colored noise, i.e., $T_{a}$ and $\tau$, can be modulated as a function of the position of nodes. In this case, the only modifications to the polygon diagram, Eq. (12), are replacing $k^{n}$ with $\prod_{i} k_{i}$ and replacing $\prod_{i} \cos \left(\theta_{i}-\alpha\right)$ with $\prod_{i} \cos \left(\theta_{i}-\alpha_{i}\right)$. Similar replacements can be performed for generic diagrams.

\section{ENERGY FLUX IN A PASSIVE SEGMENT COUPLED TO AN ACTIVE NETWORK}

A canonical setup for the study of energy transport is a passive material bar placed between the heat reservoirs held at constant temperature [Fig. 4(a)]. The generic result is that energy flows from the "hot" reservoir to the "cold" reservoir, and in the absence of a temperature difference, there can be no net energy flux through the bar.

Placing a passive material bar-in this case, three masses not influenced by any magnetic fields or active baths and connected by springs in a linear geometry-across a gap in our activated metamaterial, as illustrated in Fig. 4(a), reveals a very different behavior: Despite the absence of temperature gradients, a persistent energy flux is measured through the passive material bar. From numerical calculations, the magnitude of the energy flux decays exponentially with the bar length. In the small- $k$ limit, the flux in a (a)

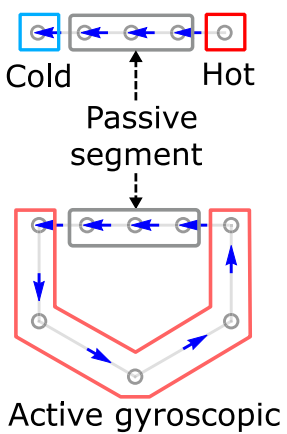

(b)

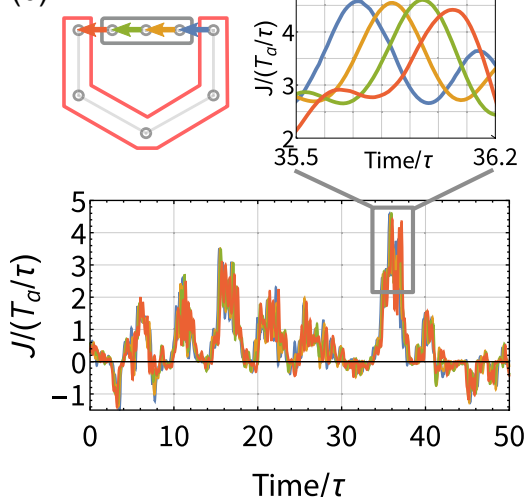

FIG. 4. Driving energy through a passive chain. (a) Conventional energy transport in a passive material (boxed in gray) with temperature differences at two ends. Similarly, the active gyroscopic network can also drive energy flows through a passive segment. (b) Instantaneous energy flux $J$ through bonds in the passive segment from a simulation. Fluxes through different bonds are colored differently. In general, $J$ is stochastic; however, during the period when $J$ is large, $J$ exhibits successive peaks in accordance with the direction of the flux. In the simulation setup, parameters for the active particles are as follows: $m, k_{g}, \gamma=0.1$, $k=10, \hat{B}, T_{a}, \tau=1$. Passive particles are constrained to $1 \mathrm{D}$, and their $\gamma, T_{a}, k_{g}$ are set to 0 . bar with $n$ sites reads $\langle J\rangle_{n}=\langle J\rangle_{1}\left(k /\left(k_{q}+m / \tau^{2}\right)\right)^{(n-1)}$, which is obtained using the diagrammatic techniques developed in Sec. V. Using numerical simulations (the method is described in Appendix H), we plot the instantaneous flux transmitted across the bonds on the passive segment in Fig. 4(b). The instantaneous flux exhibits stochastic fluctuations. Fluxes with large magnitudes are transmitted across bonds sequentially in a wavelike manner, which is reflected in the successive peaks in the instantaneous flux profile across the bonds of the passive segment [Fig. 4(b)]. The spacing between the peaks matches the sound speed in the passive chain.

This result, in combination with the results of the previous sections, shows how one can design active gyroscopic metamaterials that can act as energy pumps and support energy transport in passive materials even in the absence of any temperature gradients. Crucially, these results demonstrate how gyroscopic metamaterials can rectify nonequilibrium fluctuations. In the following section, we consider whether these rectified fluctuations, when placed in contact with a viscous fluid, can act as low Reynolds number $(R e)$ swimmers or fluid pumps [41-43].

\section{NONRECIPROCAL MOTIONS RESPONSIBLE FOR ENERGY FLUXES CAN BE USED TO GENERATE FORCES}

In this section, we show that it is possible to exploit the energy flux to rectify motions when our model systems are allowed to interact with a viscous fluid [Fig. 5(a)]. We begin by considering the motion of the three masses in the passive material bar discussed in Sec. VI and, in particular, consider the effect that their motion would have on a viscous fluid. We first do so by taking their recorded trajectories and asking whether three particles following these trajectories would "swim" in an external fluid. This calculation ignores any backaction from the fluid on the dynamics of the segment. We consider the effect of these forces in Sec. VII B and identify regimes in which this approximation is valid.

\section{A. Nonreciprocal motion as a swimming protocol}

A system of three spheres arranged in a linear configuration [Fig. 5(a)iii] provides a minimal model for low $R e$ swimming or pumping action [43]. If the lengths of the two linkages connecting the spheres, $L_{1}(t)=L+\Delta L_{1}(t)$, $L_{2}(t)=L+\Delta L_{2}(t)$, are varied according to some prescribed protocol, the time-averaged swim speed is [Eq. (12) in Ref. [43]]

$$
V_{s}=\frac{7 a}{24 L^{2}}\left\langle\Delta L_{1} \frac{d \Delta L_{2}}{d t}-\frac{d \Delta L_{1}}{d t} \Delta L_{2}\right\rangle
$$

where $a$ is the radius of the bead. Assumptions for this equation are $a / L \ll 1, \Delta L_{i} / L \ll 1$, and the total external force on the swimmer is zero. 
(a) (i)

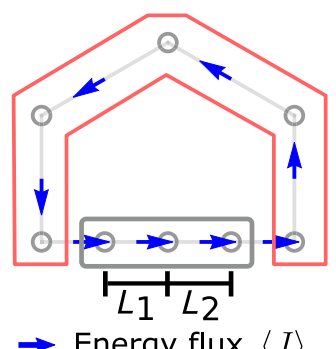

(ii)

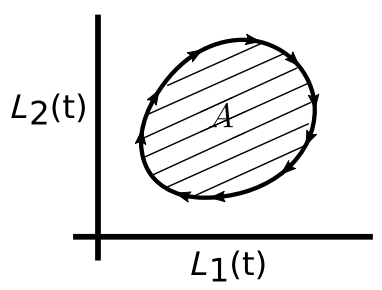

(iv)

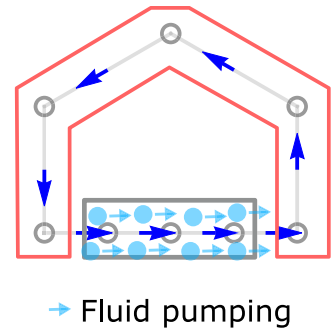

(iii)

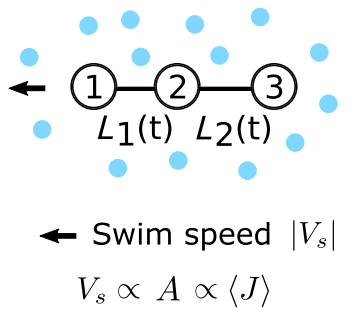

(b)

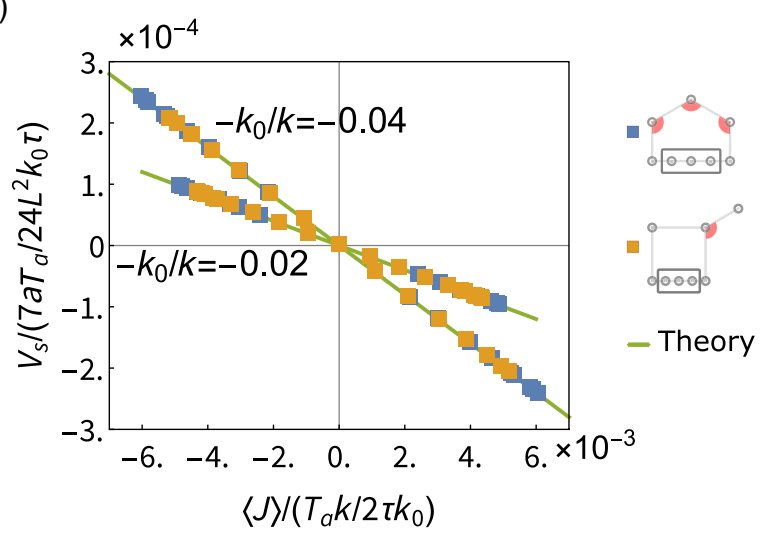

FIG. 5. Utilization of nonequilibrium gyroscopic dynamics to power swimming and pumping in low Re media. (a) The energy flux in the active gyroscopic network is accompanied by nonreciprocal motions of the particles (i,ii). The nonreciprocal motion is a schematic for illustration purposes, and the real data are much noisier. Using these nonreciprocal motions as input, a three-bead linear object can be made to swim through a low $R e$ medium (iii). Finally, by immersing the passive segment into a low $R e$ fluid, the nonreciprocal motions can be used to pump the fluid. In this manner, the energy fluxes can be rectified for locomotion and force generation (iv). (b) Swim speed $V_{s}$ is proportional to the energy flux $\langle J\rangle$ in the active network. The proportionality constant is $-k_{0} / k$, which is independent of the network geometry. The series of dots for each $-k_{0} / k$ are obtained by varying the angles labeled by red disk sectors in pentagon networks or "square + tail" networks. The parameters chosen for the numerical calculations are $m, k_{g}=0.1, \tau=1, k=5$ for $k_{0} / k=0.04$ and $k=10$ for $k_{0} / k=0.02$. For the active part, $\gamma=0.1, \hat{B}, T_{a}=1$. For the passive segment, $\gamma, \hat{B}, T_{a}=0$.

If the protocol is set to coincide with the motion of the three masses in the passive material bar that is coupled to the chiral active network discussed in Sec. VI, we find a net swim speed according to Eq. (14), proportional to the energy flux. The proportionality constant between the swim speed $V_{s}$ and the energy flux $\langle J\rangle$ can be calculated using a modified diagrammatic technique (Appendix I),

$$
\frac{V_{s}}{7 a / 24 L^{2}}=-\frac{k_{0}}{k} \frac{\langle J\rangle}{k / 2}
$$

where $k_{0}=k_{g}+m / \tau^{2}$ ( $B, \gamma=0$ for the passive segment). This result holds beyond the small- $k$ regime because all orders of diagrams are considered. Figure 5(b) and Eq. (15) together establish that one can relate the swim speed to the flux of energy in the active gyroscopic metamaterial. Similar proportionality between $V_{s}$ and $\langle J\rangle$ can be expected for other types of three-sphere swimmers, such as one where one sphere is much larger than the other two [44]. This is because the swim speed is generically proportional to the area enclosed in the $\left\{\Delta L_{1}, \Delta L_{2}\right\}$ space [45]. This area is also proportional to the energy flux $\langle J\rangle$ (Appendix I).

Thus, the nonreciprocal motion that is responsible for energy fluxes can also be used as a protocol to generate motion in a low $R e$ fluid.

\section{B. Force generation in a viscous medium using the rectified energy fluxes}

We now consider a scenario in which the passive segment connected to the active network is immersed in a fluid [Fig. 5(a)iv] and estimate the effect of the viscous backaction. Since the passive segment is tethered in place owing to the on-site potential, we focus on pumping as opposed to swimming [46] and analyze the regime for parameters where this pumping is possible. For Eq. (15) to approximately represent the pump speed as a function of the energy flux, we require that the fluid minimally perturbs the dynamics of the passive segment. Such a regime requires the dissipation rate due to the viscous fluid to be much smaller than the energy flux through the segment. This condition can be expressed as $\eta_{f} a v^{2} \ll J$, where $v$ is the characteristic velocity of a bead in the passive segment, $\eta_{f}$ is the dynamic viscosity of the fluid, and $J$ is the energy flux in the absence of the fluid. In addition, the constraint of low Reynolds number requires that $\operatorname{Re}=\rho_{f} a v / \eta_{f} \ll 1$, where $\rho_{f}$ is the fluid's density. Writing these two conditions together, the requirement reads $J \gg \eta_{f} a v^{2} \gg \rho_{f} a^{2} v^{3}$. We note that throughout this treatment, we have ignored the effect of hydrodynamic interactions between the beads on their dynamics generated from springs because it is a perturbation on the order of $a / L$.

We estimate the feasibility of our protocol using parameters at the colloidal scale, which is the scale usually considered for low $R e$ swimming. However, the magnitude of the $B$ field required to realize the above-described regime using the current setup is impractically large. Thus, it is unlikely that our metamaterials will find straightforward 
application as colloidal engines. Nonetheless, we establish that the concept of using rectification of energy to exert mechanical work is not physically impossible, motivating the search for settings in which metamaterials might find applications.

\section{CONCLUSION}

In conclusion, we have established a general set of design principles for rectifying energy and motion in nonequilibrium parity-violating metamaterials. In particular, our central results show how a combination of timereversal symmetry violation due to the geometry, interactions, and Lorentz forces in the metamaterial, and due to the nonequilibrium fluctuations of the active bath, can result in a general strategy for rectification of energy and motion. The class of tethered spring-mass network we have considered can be generalized to untethered overcoordinated elastic materials with generic interactions, which can fluctuate around its potential minimum while maintaining its shape. We note that for nonlinear models, which we do not consider in this work, it might be possible to achieve rectification without the requirement of Lorentz forces. Such extensions will be considered in future work.

\section{ACKNOWLEDGMENTS}

This work was primarily supported by National Science Foundation (NSF) Grant No. DMR-MRSEC 1420709. S. V. acknowledges support from the Sloan Foundation, start-up funds from the University of Chicago, and support from the National Science Foundation under Grant No. DMR-1848306. W. T. M. I. acknowledges support from NSF EFRI NewLAW Grant No. 1741685 and NSF Grant No. DMR 1905974.

\section{APPENDIX A: DERIVATION OF ENERGY FLUX FORMULA}

In this section, we derive the formula for energy flux, Eq. (4) in the main text. The force $F$ in this section is a generic conservative force, which does not need to be linear. We use the following strategy to determine the energy flux. First, we define the energy $E_{i}$ of particle $i$. Then, we write down an energy balance relation that expresses the infinitesimal energy change $d E_{i}$ using stochastic calculus. Finally, we collect terms in $d E_{i}$ that couple neighboring particles together and identify them as the energy transfer between particles.

The energy of particle $i$ is defined as

$$
E_{i}=\frac{1}{2} m_{i} v_{i}^{T} v_{i}+U_{i i}+\frac{1}{2} \sum_{j \neq i} U_{i j},
$$

where the first term is the kinetic energy, the second term denotes the on-site potential, and the last term is the shared spring energy between the particle and its neighbors.
We use Ito's formula to calculate $d E_{i}$. Ito's calculus provides the advantage that the stochastic terms in $d E_{i}$ vanish under time averaging. For a stochastic differential equation (SDE) of variable $X$ (vector) with drift $\mu$ (vector) and diffusion $\sigma$ (matrix),

$$
d X=\mu d t+\sigma d W
$$

where $d W$ is a vector consisting of standard Wiener processes, Ito's formula gives the SDE of function $f(X)$,

$d f(X)=\left(\left(\nabla_{X}^{T} f\right) \mu+\frac{1}{2} \operatorname{tr}\left[\sigma \sigma^{T} \nabla_{X} \nabla_{X}^{T} f\right]\right) d t+\left(\nabla_{X}^{T} f\right) \sigma d W$,

where $\nabla_{X}$ denotes the gradient with respect to $X$, the superscript $T$ denotes the transpose, and tr denotes the trace.

We begin by writing the equation of motion of our system, Eq. (1), in the form of a stochastic differential equation, Eq. (A2). We represent $N$ particles' positions by a column vector $z=\sum_{i=1}^{N}|i\rangle \otimes z_{i}$, where $|i\rangle$ denotes the $2 \mathrm{D}$ subspace of particle $i$. Similar representations are also applied to $v$ and $\eta$. Then, we get

$$
\begin{gathered}
X=\left(\begin{array}{ccc}
z & v & \eta
\end{array}\right)^{T} \\
\mu=\left(\begin{array}{c}
v \\
\frac{1}{m}\left(-\nabla_{z} U-\hat{B} A v-\gamma v+\eta\right) \\
-\frac{1}{\tau} \eta
\end{array}\right), \\
\sigma=\operatorname{diag}\left(\begin{array}{lll}
0 & 0 & \frac{\sqrt{2 \gamma T_{a}}}{\tau} I
\end{array}\right),
\end{gathered}
$$

where $U$ is the total energy of the system, $A$ is an antisymmetric matrix $A=\sum_{i}|i\rangle\langle i| \otimes A_{1}$, and $\operatorname{diag}(\cdot)$ means a block-diagonal matrix.

Now, we apply Ito's formula, Eq. (A3), to our system by associating with the function $f(X)$ the energy of particle $i$, $E_{i}(X)$. The nonzero terms in the gradient of $E_{i}$ are

$$
\begin{gathered}
\nabla_{z_{i}} E_{i}=-\left(F_{i i}+\frac{1}{2} \sum_{j} F_{j i}\right), \\
\nabla_{z_{j}} E_{i}=-\frac{1}{2} F_{i j} \\
\nabla_{v_{i}} E_{i}=m_{i} v_{i} .
\end{gathered}
$$

The term $\left(\nabla_{X}^{T} E_{i}\right) \mu$ reads

$$
\left(\nabla_{X}^{T} E_{i}\right) \mu=-\sum_{j} \frac{1}{2}\left(v_{i}+v_{j}\right)^{T} F_{i j}-\gamma v_{i}^{T} v_{i}+v_{i}^{T} \eta_{i},
$$


where we used $F_{j i}=-F_{i j}$ and $v_{i}^{T} A v_{i}=0$ in its derivation. The terms $\frac{1}{2} \operatorname{tr}\left[\sigma \sigma^{T} \nabla_{X} \nabla_{X}^{T} f\right]$ and $\nabla_{X}^{T} f$ are zero.

Finally, the energy change can be written as

$$
\begin{gathered}
d E_{i}=-\sum_{j} J_{i j} d t+h_{i} d t, \\
J_{i j}=\frac{1}{2}\left(v_{i}+v_{j}\right)^{T} F_{i j}, \\
h_{i}=-\gamma_{i} v_{i}^{T} v_{i}+v_{i}^{T} \eta_{i} .
\end{gathered}
$$

Here, $J_{i j}$ is identified as the energy transferred per unit time from particle $i$ to $j$, and $h_{i}$ is identified as the energy transferred from the bath to particle $i$.

As for the steady-state average of $J_{i j}$, we use $\left\langle d U_{i j} / d t=0\right\rangle$ and the chain rule to simplify Eq. (A12),

$$
0=v_{i}^{T} F_{j i}+v_{j}^{T} F_{i j}=-v_{i}^{T} F_{i j}+v_{j}^{T} F_{i j},
$$

and arrive at the expression

$$
\left\langle J_{i j}\right\rangle=\left\langle v_{j}^{T} F_{i j}\right\rangle .
$$

One straightforward numerical method to compute the flux $J$ is as follows. Our system is determined by the network geometry and parameters $m, k_{g}, k, \hat{B}, \gamma, \tau, T_{a}$. Given the equations of motion, Eqs. (A2) and (A4)-(A6), one can numerically solve for the covariance $C=\left\langle X X^{T}\right\rangle$ from the matrix equation $(-\mu) C+C\left(-\mu^{T}\right)=\sigma \sigma^{T}[36,37]$. Finally, the flux equation (A12), which is bilinear in $x$ and $v$, can be extracted from the covariance $C$. Numerical calculations of $\langle J\rangle$ are performed using Mathematica with a custom code [47].

\section{APPENDIX B: LINEAR RESPONSE THEORY FOR ENERGY FLUX}

Following Ref. [38], we derive the expression of the energy flux [Eqs. (7) and (10) in the main text] using a spectral linear response theory.

\section{Fourier modes for energy flux}

We define the Fourier transform (FT) of a function $f(t)$ as

$$
\begin{gathered}
\tilde{f}(\omega)=\frac{1}{t} \int_{0}^{t} d t^{\prime} f\left(t^{\prime}\right) e^{-i \omega t^{\prime}}, \quad \omega=\frac{2 \pi n}{t}, \\
f(t)=\sum_{\omega=-\infty}^{\infty} \tilde{f}(\omega) e^{i \omega t}
\end{gathered}
$$

where $n=-\infty, \ldots,-1,0,1,2, \ldots, \infty$.

The FT of the equations of motion, Eqs. (A2) and (A4)(A6), reads

$$
\begin{gathered}
\tilde{v}(\omega)=i \omega \tilde{z}(\omega), \\
\tilde{z}(\omega)=G^{+}(\omega) \tilde{\eta}(\omega), \\
\tilde{\eta}(\omega)=\frac{\sqrt{2 \gamma T_{a}}}{1+i \omega \tau} \tilde{\xi}(\omega),
\end{gathered}
$$

where $G^{+}$is the response function,

$$
G^{ \pm}(\omega)=\left[K \pm i \omega(\gamma I+\hat{B} A)-m \omega^{2} I\right]^{-1} .
$$

To make our results more general, we consider a generic noise when possible and describe it by the noise spectrum

$$
\left\langle\tilde{\eta}(\omega) \tilde{\eta}\left(\omega^{\prime}\right)^{T}\right\rangle=\frac{2 \gamma T_{a}}{t} h(\omega) I \delta_{\omega+\omega^{\prime}, 0},
$$

where $h(\omega)$ is a function that describes the shape of the spectrum. For white noise, $h(\omega)=1$. For OU colored noise, $h(\omega)=1 /\left(1+\omega^{2} \tau^{2}\right)$.

The energy flux $J_{i j}$ from Eq. (A12) can be expressed as a bilinear function in $z$ and $v$, by writing the linearized force $F$ in terms of $z$,

$$
\begin{gathered}
J_{i j}=k v^{T} A^{J} z, \\
A^{J} \equiv \frac{1}{2}\left(|i\rangle\left\langle i\left|\otimes e_{i j} e_{i j}^{T}+\right| i\right\rangle\langle j| \otimes e_{i j} e_{j i}^{T}\right. \\
\left.+|j\rangle\left\langle i\left|\otimes\left(-e_{j i} e_{i j}^{T}\right)+\right| j\right\rangle\langle j| \otimes\left(-e_{j i} e_{j i}^{T}\right)\right) .
\end{gathered}
$$

This bilinear form enables us to write the time integral of the energy flux $Q=\int_{0}^{t} d t^{\prime} J\left(t^{\prime}\right)$ as a sum of Fourier modes $\tilde{q}_{\omega}$ using Parseval's theorem,

$$
\begin{gathered}
Q=t \sum_{\omega=-\infty}^{\infty} \tilde{q}_{\omega}, \\
q_{\omega}=k \tilde{v}^{T} A^{J} \tilde{z}^{*}=i \omega k \tilde{\eta}^{T} G^{+T} A^{J} G^{-} \tilde{\eta}^{*},
\end{gathered}
$$

where the superscript $*$ denotes the complex conjugate.

Pairing $\tilde{q}_{\omega}$ and its conjugate $\tilde{q}_{-\omega}$ gives a real function, which would be beneficial for subsequent derivations:

$$
\begin{gathered}
Q=t \sum_{\omega=2 \pi / t}^{\infty}\left(\tilde{q}_{\omega}+\tilde{q}_{-\omega}\right), \\
\tilde{q}_{\omega}+\tilde{q}_{-\omega}=\tilde{\eta}(\omega)^{T} A_{\omega}^{q} \tilde{\eta}(\omega)^{*} \\
A_{\omega}^{q}=-i \omega k G^{+}(\omega)^{T} A^{\text {as }} G^{-}(\omega), \\
A^{\text {as }}=-\left(A^{J}-A^{J^{T}}\right) \\
=-|i\rangle\left\langle j\left|\otimes e_{i j} e_{j i}^{T}+\right| j\right\rangle\langle i| \otimes e_{j i} e_{i j}^{T} .
\end{gathered}
$$


Averaging $\tilde{q}_{\omega}+\tilde{q}_{-\omega}$ over the noise $\tilde{\eta}(\omega)$, we get

$$
\left\langle\tilde{q}_{\omega}+\tilde{q}_{-\omega}\right\rangle=\frac{2 \gamma T_{a}}{t} h(\omega) \operatorname{tr} A_{\omega}^{q} .
$$

\section{Integrating over the Fourier modes}

In a long time limit, the sum can be approximated by an integral

$$
\frac{1}{t} \sum_{\omega=2 \pi / t}^{\infty}=\frac{1}{2 t} \sum_{\omega=-\infty}^{\infty} \frac{t \Delta \omega}{2 \pi} \approx \frac{1}{4 \pi} \int_{-\infty}^{\infty} d \omega .
$$

Equations (B12) and (B17) can then be turned to an integral expression of the flux,

$$
\langle J\rangle=\lim _{t \rightarrow \infty} \frac{\langle Q\rangle}{t}=\frac{\gamma T_{a}}{2 \pi} \int_{-\infty}^{\infty} d \omega h(\omega) \operatorname{tr} A_{\omega}^{q} .
$$

In the next steps, we simplify this integral with the help of the property from Ref. [38],

$$
G^{-}(\omega)-G^{+}(\omega)^{T}=2 i \omega \gamma G^{-}(\omega) G^{+}(\omega)^{T} .
$$

Using this property, the trace of $A_{\omega}^{q}$ becomes

$$
\begin{aligned}
\operatorname{tr} A_{\omega}^{q} & =-i \omega k \operatorname{tr} G^{+T} A^{\mathrm{as}} G^{-} \\
& =-i \omega k \frac{1}{2 i \omega \gamma} \operatorname{tr}\left(G^{-}-G^{+T}\right) A^{\text {as }} \\
& =-\frac{k}{\gamma} \operatorname{Re} \operatorname{tr} G^{+} A^{\mathrm{as}} .
\end{aligned}
$$

Plugging this trace into Eq. (B19), we get the integral form for the flux equation (7),

$$
\langle J\rangle=-\frac{T_{a} k}{2 \pi} \int_{-\infty}^{\infty} d \omega h(\omega) \operatorname{Re} \operatorname{tr} G^{+} A^{\mathrm{as}} .
$$

Since $\operatorname{Im} G^{+}(-\omega)=-\operatorname{Im} G^{+}(\omega), h(\omega) \operatorname{Im} \operatorname{tr} G^{+} A^{\text {as }}$ is an odd function of $\omega$, and its line integral vanishes,

$$
\langle J\rangle=-\frac{T_{a} k}{2 \pi} \int_{-\infty}^{\infty} d \omega h(\omega) \operatorname{tr} G^{+} A^{\text {as }} .
$$

The difference between this expression and Eq. (B22) is that the integrand of Eq. (B22) is real at all $\omega$ 's, whereas the integrand of Eq. (B22) is complex. We adopt Eq. (B22) in the main text, so its integrand can be interpreted as the flux of Fourier modes.

We now consider the specific OU colored noise, $h(\omega)=1 /\left(1+\omega^{2} \tau^{2}\right)$. In this case, the integral in Eq. (B23) can be calculated using the residue theorem. The integrand vanishes at $\omega \rightarrow \infty$, so the line integral can be converted to a contour integral along the counterclockwise semicircle $R$ in the lower half-plane,

$$
\langle J\rangle=\frac{T_{a} k}{2 \pi} \oint_{R} d \omega \frac{\operatorname{tr} G^{+} A^{\mathrm{as}}}{1+\omega^{2} \tau^{2}} .
$$

The $\operatorname{tr} G^{+} A^{\text {as }}$ part in the integrand has no pole in the lower half-plane because $G^{+}$is a linear response function, and it satisfies causality. However, the noise correlation $\tau$ introduces one pole at $\omega=-i / \tau$; thus, the contour integral can be evaluated as

$$
\langle J\rangle=-\frac{T_{a} k}{2 \tau} \operatorname{tr} G^{+}\left(-\frac{i}{\tau}\right) A^{\mathrm{as}},
$$

and the response function at $\omega=-i / \tau$ reads

$$
G^{+}\left(-\frac{i}{\tau}\right)=\left[K+\left(\frac{\gamma}{\tau}+\frac{m}{\tau^{2}}\right) I+\frac{\hat{B}}{\tau} A\right]^{-1} .
$$

In theory, Eq. (B25) provides the analytical solution of the flux because the inverse matrix, Eq. (B26), can be expressed analytically. In practice, analytical solutions can be easily calculated for small networks but are impractical for large networks.

Nevertheless, some general properties of the flux can be obtained from Eq. (B25) after some algebra. For a network with only horizontal and vertical bonds [Fig. 1(b)], all fluxes are zero. For two networks whose slanted bonds have opposite angles [top and bottom hexagons in Fig. 1(b)], their fluxes are opposite. Changing $B$ to $-B$ would change the flux $J$ to $-J$.

\section{Moments of the energy transport}

Higher-order moments of the integrated energy flux $Q=$ $\int_{0}^{t} d t^{\prime} J\left(t^{\prime}\right)$ can also be calculated following methods in Ref. [38]. We define the moment-generating function of $Q$ as $Z(\lambda)=\left\langle e^{\lambda Q}\right\rangle$. In the long time limit, $Z(\lambda)$ can be expressed in the large-deviation form [38], $Z(\lambda)=e^{t \mu(\lambda)}$, where $\mu(\lambda)$ is called the rate function. For our system, $Z(\lambda)$ can be calculated through Gaussian integrals. The resulting $\mu(\lambda)$ reads

$\mu(\lambda)=-\frac{1}{4 \pi} \int_{-\infty}^{\infty} d \omega \operatorname{tr} \ln \left[I+\lambda 2 \gamma T_{a} h(\omega) A^{q}(\omega)\right]$,

where $A^{q}(\omega)$ is defined in Eq. (B14).

The first moment $\langle Q\rangle$ reads

$$
\begin{aligned}
\langle Q\rangle & =\left.\frac{\partial Z(\lambda)}{\partial(-\lambda)}\right|_{\lambda \rightarrow 0}=-t \partial_{\lambda} \mu(\lambda) \\
& =\frac{t \gamma T_{a}}{2 \pi} \int_{-\infty}^{\infty} d \omega h(\omega) \operatorname{tr} A^{q}(\omega),
\end{aligned}
$$

which is consistent with the expression in the main text, Eq. (7). 
The fluctuation $\left\langle(\delta Q)^{2}\right\rangle=\left\langle Q^{2}\right\rangle-\langle Q\rangle^{2}$ reads

$$
\begin{aligned}
\left\langle(\delta Q)^{2}\right\rangle & =\left.\frac{\partial^{2} Z(\lambda)}{\partial(-\lambda)^{2}}\right|_{\lambda \rightarrow 0}-\langle Q\rangle^{2}=t \partial_{\lambda}^{2} \mu(\lambda) \\
& =\frac{t\left(\gamma T_{a}\right)^{2}}{\pi} \int_{-\infty}^{\infty} d \omega h(\omega)^{2} \operatorname{tr}\left(A^{q}(\omega)^{2}\right) .
\end{aligned}
$$

Plugging in the expression for $A^{q}(\omega)$, Eq. (B14), and using the property in Eq. (B20), $\left\langle(\delta Q)^{2}\right\rangle$ simplifies to

$$
\begin{aligned}
\left\langle(\delta Q)^{2}\right\rangle= & \frac{t\left(T_{a} k\right)^{2}}{4 \pi} \int_{-\infty}^{\infty} d \omega h(\omega)^{2} \operatorname{tr}\{ \\
& \left.\left(G^{-}-G^{+T}\right) A^{\text {as }}\left(G^{-}-G^{+T}\right) A^{\text {as }}\right\} .
\end{aligned}
$$

In the noninteracting $(k \rightarrow 0)$ limit, the following quantity tends to a nonvanishing constant,

$$
\begin{gathered}
\lim _{k \rightarrow 0} \frac{\left\langle(\delta Q)^{2}\right\rangle}{k^{2}}=-\frac{t T_{a}^{2}}{2 \pi} \int_{-\infty}^{\infty} d \omega h(\omega)^{2}(g(\omega)-g(-\omega))^{2}, \\
g(\omega)=\frac{k_{g}+i \omega \gamma-m \omega^{2}}{\left(k_{g}+i \omega \gamma-m \omega^{2}\right)^{2}-(\omega \hat{B})^{2}} .
\end{gathered}
$$

This limiting value is independent of the network geometry and thus reflects inherent fluctuations for energy fluxes between two bonded sites regardless of the rest of the network.

\section{APPENDIX C: DERIVATION OF KIRCHOFF'S LAW}

The derivation of Kirchoff's law is similar to the derivation of the energy flux, except that we use the energy from the bath to the particle $h_{i}$ in Eq. (A13) instead of $J_{i j}$ in Eq. (A12).

Following the procedure in Appendix $\mathrm{B}$ from Eqs. (B12)-(B19), we arrive at an integral expression for $\left\langle h_{i}\right\rangle$ with a different $A_{\omega}^{q}$,

$$
\begin{gathered}
\left\langle h_{i}\right\rangle=\frac{\gamma T_{a}}{2 \pi} \int_{-\infty}^{\infty} d \omega h(\omega) \operatorname{tr} A_{\omega}^{q}, \\
A_{\omega}^{q}=i \omega\left(G^{+T} \rho_{i}-\rho_{i} G^{-}\right)-2 \gamma \omega^{2} G^{+T} \rho_{i} G^{-}, \\
\rho_{i} \equiv|i\rangle\langle i| .
\end{gathered}
$$

Here, the noise is kept generic.

Using the property of $G^{ \pm}$in Eq. (B20), we get

$$
\operatorname{tr}\left(G^{+T} \rho_{i}-\rho_{i} G^{-}\right)=-2 i \omega \gamma \operatorname{tr} G^{+T} \rho_{i} G^{-} .
$$

The trace of $A_{\omega}^{q}$ then vanishes, $\operatorname{tr} A_{\omega}^{q}=i \omega \operatorname{tr}\left(G^{+T} \rho_{i}-\rho_{i} G^{-}\right)-\operatorname{tr} 2 \gamma \omega^{2} G^{+T} \rho_{i} G^{-}=0$.

From Eq. $(\mathrm{C} 1),\left\langle h_{i}\right\rangle$ is also zero, so on average there is no net energy exchange between the particle and the bath. Because the average change of $E_{i}$ is zero, and $\left\langle\dot{E}_{i}\right\rangle=$ $-\sum_{j}\left\langle J_{i j}\right\rangle+\left\langle h_{i}\right\rangle$, we obtain Kirchoff's law,

$$
-\sum_{j}\left\langle J_{i j}\right\rangle=\sum_{j}\left\langle J_{j i}\right\rangle=0 .
$$

\section{APPENDIX D: A HARADA-SASA RELATION}

We present a Harada-Sasa relation [39] for our active gyroscopic networks, which shows a connection between the fluctuation-response breaking and an entropy production rate. For the calculation of the entropy production rate, there have been debates in the literature on what the reverse process should be, especially for systems in the presence of Lorentz-like forces and systems with active particles [48-51]. Here, we simply choose some prescriptions for the reverse process and show that such a choice is consistent with the Harada-Sasa relation.

In this section, our system is slightly modified by adding additional white noise $\xi_{w}$ with correlation $\left\langle\xi_{w}(t) \xi_{w}\left(t^{\prime}\right)^{T}\right\rangle=$ $2 \gamma T_{a} I \delta\left(t-t^{\prime}\right)$, and the white noise is uncorrelated with the color noise $\eta$. The additional white noise simplifies the discussion of the entropy production rate; at the same time, it does not affect the energy flux. In order to directly compare our result with the Harada-Sasa relation, we define the Fourier transform as $\tilde{f}(\omega)=\int_{-\infty}^{\infty} d t f(t) e^{-i \omega t}$. This definition is slightly different from the rest of the paper, and it is restricted to this section. We also keep the colored noise generic, $\left\langle\tilde{\eta}(\omega) \tilde{\eta}\left(\omega^{\prime}\right)^{* T}\right\rangle=4 \pi \gamma T_{a} h(\omega) \delta\left(\omega+\omega^{\prime}\right)$.

The resulting Harada-Sasa relation reads

$$
\dot{s}=\frac{\gamma}{T_{a}} \int \frac{d \omega}{2 \pi} \operatorname{tr}\left[\tilde{C}(\omega)-2 T_{a} \operatorname{Re} \tilde{R}(\omega)\right],
$$

where $\dot{s}$ is the entropy production rate, $\tilde{C}(\omega)$ is the velocity correlation, and $\tilde{R}(\omega)$ is the velocity response.

\section{Fluctuation-response breaking}

The equation of motion with additional white noise reads

$$
m \dot{v}=-K z-\hat{B} A v-\gamma v+\eta+\xi_{w} .
$$

The FT of the equation of motion leads to

$$
\tilde{v}=i \omega G^{+}(\omega)\left(\tilde{\eta}+\tilde{\xi}_{w}\right),
$$

where $G^{+}(\omega)$ is the same as in Eq. (6). 
The response matrix for the velocity reads

$$
\tilde{R}(\omega)=i \omega G^{+}(\omega) .
$$

The velocity correlation in the time domain is defined as $C(t)=\left\langle v(t) v(0)^{T}\right\rangle$. To extract a simplified $\tilde{C}$, we use the Fourier transform $\tilde{v}$ [Eq. (D3)], plug in the noise correlation, and utilize the property of $G^{+}$[Eq. (B20)]; then, we get

$$
\tilde{C}(\omega)=-2 \omega T_{a}(1+h(\omega)) \operatorname{Im} G^{+} .
$$

Combining Eqs. (D4) and (D5), the breaking of the fluctuation response reads

$$
\operatorname{tr}\left[\tilde{C}-2 T_{a} \operatorname{Re} \tilde{R}\right]=2 i \omega T_{a} h(\omega) \operatorname{tr} i \operatorname{Im} G^{+} .
$$

To simplify its integral, we use the property that $\operatorname{Re} G^{+}(\omega)=\operatorname{Re} G^{+}(-\omega)$, and $\omega h(\omega) \operatorname{Re} G^{+}(\omega)$ is an odd function in $\omega$. So $\int d \omega \omega h(\omega) i \operatorname{Im} G^{+}=\int d \omega \omega h(\omega) G^{+}$, and the result reads

$$
\int \frac{d \omega}{2 \pi} \operatorname{tr}\left[\tilde{C}-2 T_{a} \operatorname{Re} \tilde{R}\right]=\int \frac{d \omega}{2 \pi} 2 i \omega T_{a} h(\omega) \operatorname{tr} G^{+} .
$$

\section{Entropy production rate}

Following the framework of stochastic thermodynamics [1], the entropy production rate is given by

$$
\dot{s}=\lim _{t \rightarrow \infty} \frac{1}{t} \log \frac{\mathcal{P}}{\mathcal{P}^{\dagger}},
$$

where $\mathcal{P}$ and $\mathcal{P}^{\dagger}$ are the probabilities of the forward trajectory and the reverse trajectory.

The probability of the trajectory is given by the OnsagerMachlup path integral [52],

$$
\begin{gathered}
\mathcal{P} \propto e^{-\mathcal{A}} \prod_{t^{\prime}} \delta\left(\partial_{t^{\prime}} z-v\right), \\
\mathcal{A}=\int_{0}^{t} d t^{\prime} \frac{m^{2}}{4 \gamma T_{a}}\left|\partial_{t^{\prime}} v+\frac{\gamma}{m} v-\frac{1}{m} F_{p}\right|^{2}, \\
F_{p} \equiv-K z-\hat{B} A v+\eta .
\end{gathered}
$$

For the conjugation trajectory, we choose the prescription that the $B$ field flips sign, $B^{\dagger}=-B$ [49], and $\eta^{\dagger}=\eta$ for the colored noise. With these prescriptions, $\mathcal{P}^{\dagger}$, and consequently $\dot{s}$ from Eq. (D8), can be calculated,

$$
\dot{s}=-\frac{1}{T_{a}}\left\langle v^{T}\left(m \dot{v}-F_{p}\right)\right\rangle .
$$

We plug in the expression for $F_{p}$, find that many terms vanish, and get

$$
\dot{s}=\frac{1}{T_{a}}\left\langle v^{T} \eta\right\rangle=\frac{1}{T_{a}} \operatorname{tr}\left\langle v \eta^{T}\right\rangle
$$

Expressing $v$ in terms of the response function,

$$
\dot{s}=\frac{1}{T_{a}} \int \frac{d \omega}{2 \pi} \operatorname{tr} i \omega G^{+}(\omega) 2 \gamma T_{a} h(\omega) .
$$

Compare the entropy production rate, Eq. (D14), and the fluctuation-response breaking, Eq. (D7). We arrive at a Harada-Sasa relation, Eq. (D1).

Note that if we choose the prescription $B^{\dagger}=B$, the calculated $\dot{s}$ would be different; consequently, there would be no Harada-Sasa-like relation. The discrepancy between prescriptions $B^{\dagger}=-B$ and $B^{\dagger}=B$ seems to suggest that flipping the $B$ field should give the appropriate prescription from the perspective of relating the fluctuation-response breaking to an entropy production rate.

\section{APPENDIX E: CONNECTION OF ENERGY FLUX IN ACTIVE NETWORKS TO EIGENMODES IN ISOLATED GYROSCOPIC NETWORKS}

\section{The connection and its application to honeycomb lattices}

If excluding the active bath, our model is identical to the isolated systems studied in Refs. [29-32]. We would like to build a connection between our energy flux in the active system and eigenmodes in those studies. In this subsection, we show that the energy flux in active gyroscopic networks can be expanded as a weighted sum over eigenmodes of a reference isolated system, Eq. (E2). Then, we apply this result to the honeycomb lattice as an example.

The Fourier analysis from Sec. IV in the main text is not suitable for this connection because Fourier modes and eigenmodes are related only at small $\gamma$ 's [Figs. 6(a) and 6(b)], but they become dissimilar at larger $\gamma$ 's [Figs. 6(a) and 6(c)]. The underlying discrepancy between Fourier modes and eigenmodes comes from the fact that eigenmodes are a natural basis for the isolated network, whereas Fourier modes contain an extra factor of friction or damping. In addition to this extra factor $\gamma$, the active system also has extra factors of $m$ and $\tau$. The factor $m$ comes from the order of dynamics: The active system is second order in time, while the gyroscopic dynamics in Ref. [29] is first order, which corresponds to the $m \rightarrow 0$ limit.

Our starting point is Eq. (10). The key observation is that, in the function $G^{+}(-i / \tau)$ from the equation, $\gamma, m, \tau$ are not independent factors. Rather, they act collectively through

$$
k_{g, \tau} \equiv k_{g}+\frac{\gamma}{\tau}+\frac{m}{\tau^{2}}
$$

In effect, the extra factors $m, \gamma, \tau$ only add a modification to $k_{g}$. Following these ideas, we imagine a reference isolated 
(a) Eigenmode

(b) Fourier mode small damping

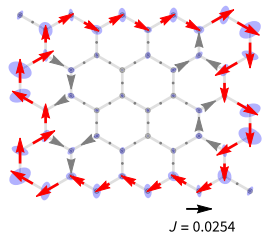

(c) Fourier mode larger damping

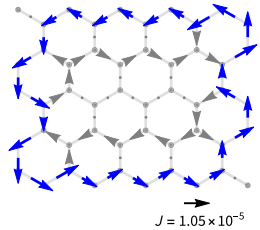

FIG. 6. Comparison between a boundary-localized eigenmode of the undamped isolated network and the Fourier modes of the damped network at the same frequency. First-order dynamics (by setting $m=0$ ) are used. Numerical calculations are performed with all other parameters set to 1. (a) Eigenmode of undamped gyroscopic system. For the frequency chosen, the eigenmode is localized on the boundary. Blue disks represent the orbit of particles. (b) The Fourier mode of the damped variant of our model at small $\gamma(\gamma=0.001)$ resembles the eigenmode. (c) The Fourier mode at larger $\gamma(\gamma=1)$ is no longer close to the eigenmode.

system with modified on-site spring constant $k_{g, \tau}$. The flux $\langle J\rangle$ in the active system can be written as a weighted sum of the flux of each eigenmode $J_{\omega_{e}}^{\text {eig }}$ in the reference system (see Appendix E 2 for the derivation),

$$
\langle J\rangle=\sum_{\omega_{e}} \frac{1}{1+\omega_{e}^{2} \tau^{2}} J_{\omega_{e}}^{\mathrm{eig}} .
$$

Here, $\omega_{e}$ is the discrete eigenfrequency of the reference system, not to be confused with the continuous Fourier frequency $\omega$. The amplitude of the eigenmode is set such that its energy is $T_{a}$, and $J_{\omega_{e}}^{\mathrm{eig}}$ is the time-averaged energy flux.

A related result is a "sum rule"; namely, the unweighted sum of all modes is zero,

$$
\sum_{\omega_{e}} J_{\omega_{e}}^{\mathrm{eig}}=0 .
$$

This sum rule can be derived from direct calculations (see Appendix E 2).

From this eigenmode decomposition, the discussion of time-reversal symmetry in the isolated system [29] immediately carries over to the active system. For network geometries that satisfy time-reversal symmetries, the energy flux of eigenmodes is zero. Thus, through Eq. (E2), the flux in an active system is also zero. This result can alternatively be obtained from Eq. (10) through some linear algebra.

As an application, we analyze the flux in the honeycomb network using the eigenmode decomposition, Eq. (E2), and the sum rule, Eq. (E3). The flux pattern in the active honeycomb network displays CCW flux localized on the boundary [Fig. 1(b)]. This localization is reminiscent of the edge mode in Ref. [29] [Fig. 6(b)]; however, their directions are opposite. From the decomposition, Eq. (E2), the edge

(a)

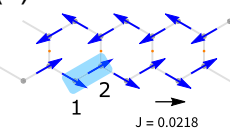

(d)

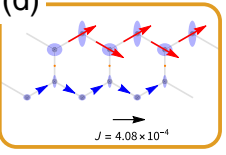

(e)
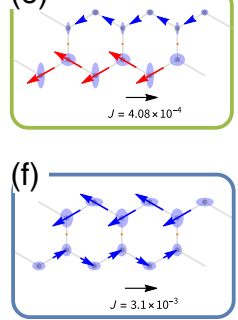

(b)

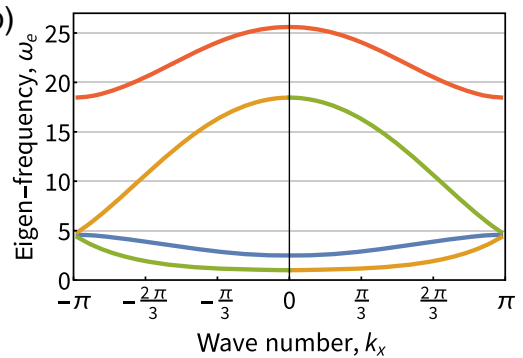

(c)

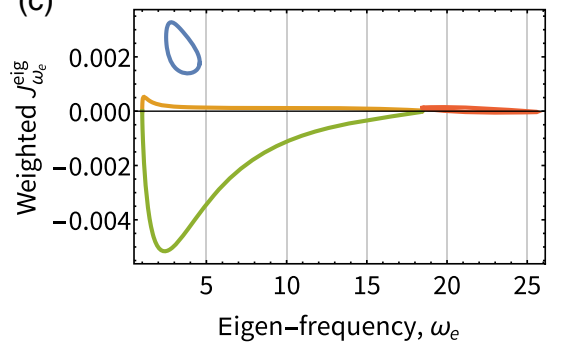

FIG. 7. Using the eigenmode decomposition, we explain how the flux in the honeycomb network is $\mathrm{CCW}$, even though its edge modes contribute to CW fluxes. (a) Network used for calculation, which consists of one row of hexagons (51 unit cells) and has a periodic boundary in the $x$ direction. The parameters are $k_{g, \tau}=1$, $k=10$, with others being 1 . (b) Band structure of the network (marked with different colors). The yellow or green band contains $\mathrm{CW}$ flux localized on the top or bottom edge [an example mode is shown in panel (d) or (e)]. The blue band contains bulk modes with CCW flux [also see panel (f)]. (c) Weighted flux $J_{\omega_{e}}^{\text {eig }}$ from 1 to 2 [marked in panel (a)] of the four bands. The total flux in the green band with $\mathrm{CW}$ edge modes and that in the blue band with CCW bulk modes are -0.106 and 0.115 , respectively. As a result, the net flux is CCW.

modes should contribute a large $\mathrm{CW}$ flux in the active system, but somewhat surprisingly, the net flux is CCW. To better analyze the contribution from each eigenmode, we look at a simple honeycomb lattice with only one layer [Fig. 7(a)]. This lattice has four bands [Fig. 7(b)], two bulk bands (blue, red) and two edge bands (green, yellow). The weighted flux of each band is plotted in Fig. 7(c). We see that the $\mathrm{CW}$ edge band contributes a large $\mathrm{CW}$ flux [green curve in Fig. 7(c)]; however, due to the sum rule, the unweighted sum of other bands has to be CCW. In the honeycomb lattice, many of the CCW fluxes are contained in the lower bulk band [blue curve in Fig. 7(c) and example mode in Fig. 7(f)]. When the flux gets weighted, the CCW flux from the lower bulk band outweighs the $\mathrm{CW}$ flux from the edge modes; the other two bands [yellow and red curves in Fig. 7(c)] also contribute to the CCW flux, although the contribution is relatively small. As a result, the net flux is CCW, which is opposite to the flux of the edge mode.

\section{Derivation of the connection}

To derive the eigenmode decomposition, Eq. (E2), we first look at the reference isolated system and write down its 
eigenmodes and time-averaged energy flux, Eq. (E10). Then, we turn to the active system and decompose the flux using the eigenmodes to get Eq. (E13). Finally, we show that the fluxes from these two sides are actually related in Eq. (E17). Lastly, we also derive the sum rule, Eq. (E19).

\section{a. Energy flux in the reference isolated system}

The reference isolated system has first-order gyroscopic dynamics as in Ref. [29]. In the setup with Lorentz force, the dynamical equation can be obtained by setting the mass to zero and replacing the force matrix $K$ by $K^{\tau} \equiv K+$ $\left[(\gamma / \tau)+\left(m / \tau^{2}\right) I\right.$

$$
\dot{z}=\frac{1}{\hat{B}} A K^{\tau} z
$$

Following Ref. [29], we convert to a complex representation with $z^{c} \equiv(x+i y x-i y)^{T}$,

$$
i \ddot{z}^{c}=\Omega z^{c}, \quad K^{\tau}=i \hat{B} A O^{-1} \Omega O,
$$

where $O, O^{-1}$ are the transformations between $z$ and $z^{c} z^{c}=O z, z=O^{-1} z^{c}$.

Writing the eigenvalue problem as

$$
\Omega u_{\omega_{e}}=\omega_{e} u_{\omega_{e}},
$$

the eigenmode with eigenfrequency $\omega_{e}$ reads

$$
z_{\omega_{e}}^{c}(t)=\left(u_{\omega_{e}} e^{-i \omega_{e} t}+u_{-\omega_{e}} e^{i \omega_{e} t}\right) z_{0},
$$

where $z_{0}$ is the amplitude, which will be specified shortly. The eigenmode needs a combination of $\omega_{e}$ and $-\omega_{e}$ to ensure that the motion of $x$ and $y$ is real-valued. Mathematically, this combination is possible because of a symmetry in this eigenvalue problem; when there is $\omega_{e}$, there is also a solution $-\omega_{e}$ with

$$
u_{-\omega_{e}}=\left(\begin{array}{cc}
0 & I \\
I & 0
\end{array}\right) u_{\omega_{e}}^{*} .
$$

A related property we will need later is that the left eigenvector $v_{\omega_{e}}$ can be expressed as

$$
v_{\omega_{e}}=c_{\omega_{e}}\left(\begin{array}{cc}
-I & 0 \\
0 & I
\end{array}\right) u_{\omega_{e}},
$$

where $c_{\omega_{e}}$ is a real prefactor, to ensure normalization $v_{\omega_{e}}^{T} u_{\omega_{e}}=1$. If there are degenerate eigenvectors (like $\left.v_{\omega_{e}}^{1}, v_{\omega_{e}}^{2}, \ldots\right)$, we choose an orthonormal basis set, i.e., $v_{\omega_{e}}^{i, T} u_{\omega_{e}}^{j}=0$ for $i \neq j$. With the introduction of $c_{\omega_{e}}$, we now set the amplitude to $z_{0}^{2}=-2 c_{\omega_{e}} T_{a} / \omega_{e} \hat{B}$, such that the energy of the eigenmode is $T_{a}$.
The instantaneous energy flux $J_{\omega_{e}}$ of mode $z_{\omega_{e}}^{c}$ reads

$$
\begin{aligned}
J_{\omega_{e}} & =\left(O^{-1} v_{\omega_{e}}^{c}\right)^{T} A^{J} O^{-1} z_{\omega_{e}}^{c} \\
& =\operatorname{tr} O^{-1, T} A^{J} O^{-1} z_{\omega_{e}}^{c} v_{\omega_{e}}^{c T},
\end{aligned}
$$

where $A^{J}$ has been defined in Eq. (B9). The factor $z_{\omega_{e}}^{c} v_{\omega_{e}}^{c T}$ can be computed using Eq. (E7). When averaging over periods of oscillation, terms containing $e^{ \pm 2 i \omega_{e} t}$ vanish, and we get

$$
\overline{z_{\omega_{e}}^{c} v_{\omega_{e}}^{c T}}=i \omega_{e}\left(u_{\omega_{e}} u_{-\omega_{e}}^{T}-u_{-\omega_{e}} u_{\omega_{e}}^{T}\right) z_{0}^{2}
$$

Plugging in $z_{0}^{2}=-2 c_{\omega_{e}} T_{a} / \omega_{e} \hat{B}$, the time-averaged flux of the eigenmode $J_{\omega_{e}}^{\mathrm{eig}}$ reads

$$
J_{\omega_{e}}^{\mathrm{eig}}=-\frac{2 T_{a} k}{\hat{B}} i c_{\omega_{e}} \operatorname{tr} O^{-1, T} A^{J} O^{-1}\left(u_{\omega_{e}} u_{-\omega_{e}}^{T}-u_{-\omega_{e}} u_{\omega_{e}}^{T}\right) .
$$

\section{b. Energy flux of the active system}

Now, we turn to the active system, and the starting point is Eq. (10). We need to decompose $G^{\tau} \equiv G^{+}(-i / \tau)$ into modes as below. We use $u_{\omega_{e}}$ and $v_{\omega_{e}}$ to denote the right and left eigenvectors for matrix $\Omega$ at eigenfrequency $\omega_{e}$, respectively:

$$
G^{\tau}=\frac{i}{\hat{B}} O^{-1}\left(\Omega-\frac{i}{\tau} I\right)^{-1} O A,
$$

$$
\begin{aligned}
\left(\Omega-\frac{i}{\tau} I\right)^{-1} & =\sum_{\omega_{e}>0} \frac{i \tau}{1+\omega_{e}^{2} \tau^{2}}\left(u_{\omega_{e}} v_{\omega_{e}}^{T}+u_{-\omega_{e}} v_{-\omega_{e}}^{T}\right) \\
& +\sum_{\omega_{e}>0} \frac{\omega_{e} \tau^{2}}{1+\omega_{e}^{2} \tau^{2}}\left(u_{\omega_{e}} v_{\omega_{e}}^{T}-u_{-\omega_{e}} v_{-\omega_{e}}^{T}\right) .
\end{aligned}
$$

When plugging $G^{\tau}$ into Eq. (10), the contribution from the second term in $[\Omega-(i / \tau) I]^{-1}$ vanishes, $\operatorname{tr} O A A^{\text {as }} O^{-1} \times$ $\left(u_{\omega_{e}} v_{\omega_{e}}^{T}-u_{-\omega_{e}} v_{-\omega_{e}}^{T}\right)=0$. We arrive at a decomposition of the flux in active system into eigenmodes in a reference isolated system,

$$
\begin{gathered}
\langle J\rangle=\sum_{\omega_{e}}\langle J\rangle_{\omega_{e}}, \\
\langle J\rangle_{\omega_{e}} \equiv \frac{T_{a} k / 2 \hat{B}}{1+\omega_{e}^{2} \tau^{2}} \operatorname{tr} O A A^{\text {as }} O^{-1}\left(u_{\omega_{e}} v_{\omega_{e}}^{T}+u_{-\omega_{e}} v_{-\omega_{e}}^{T}\right) .
\end{gathered}
$$

\section{c. Connection between isolated system and active system}

Now, we need to find the connection between these two fluxes, $\langle J\rangle_{\omega_{e}}$ in Eq. (E14) and $J_{\omega_{e}}^{\text {eig }}$ in Eq. (E10). We write 
$J_{\omega_{e}}^{\text {eig }}$ in a form that looks similar to $\langle J\rangle_{\omega_{e}}$. Converting $A^{J}$ to $A^{\text {as }}$ using $A^{\text {as }}=-\left(A^{J}-A^{J T}\right)$ [Eq. (B15)], and $u_{\omega_{e}}$ to $v_{\omega_{e}}$ using

$$
v_{\omega_{e}}=c_{\omega_{e}}\left(\begin{array}{cc}
-I & 0 \\
0 & I
\end{array}\right) u_{\omega_{e}}
$$

we get

$J_{\omega_{e}}^{\mathrm{eig}}=-\frac{i T_{a} k}{\hat{B}} \operatorname{tr} A O^{-1, T} A^{\mathrm{as}} O^{-1}\left(u_{\omega_{e}} v_{\omega_{e}}^{T}+u_{-\omega_{e}} v_{-\omega_{e}}^{T}\right)$.

From direct calculation, $A O^{-1, T}=(i / 2) O A$, and $J_{\omega_{e}}^{\mathrm{eig}}$ becomes the same as $\langle J\rangle_{\omega_{e}}$ apart from a factor

$$
J_{\omega_{e}}^{\mathrm{eig}}=\frac{T_{a} k}{2 \hat{B}} \operatorname{tr} O A A^{\mathrm{as}} O^{-1}\left(u_{\omega_{e}} v_{\omega_{e}}^{T}+u_{-\omega_{e}} v_{-\omega_{e}}^{T}\right) .
$$

Comparing Eq. (E16) with Eq. (E14), we arrive at the connection between the flux from the active system and that from the reference isolated system,

$$
\langle J\rangle_{\omega_{e}}=\frac{1}{1+\omega_{e}^{2} \tau^{2}} J_{\omega_{e}}^{\mathrm{eig}} .
$$

Additionally, the unweighted sum reads

$$
\sum_{\omega_{e}} J_{\omega_{e}}^{\mathrm{eig}}=\frac{T_{a} k}{2 \hat{B}} \operatorname{tr}\left[O A A^{\mathrm{as}} O^{-1} U V^{T}\right],
$$

where $U$ is the collection of all right eigenvectors $U=\left(u_{\omega_{e}, 1} u_{\omega_{e}, 2} \cdots\right)$, and likewise for $V$. Since $U V^{T}=$ $I$ from orthonormality, the unweighted sum vanishes,

$$
\sum_{\omega_{e}} J_{\omega_{e}}^{\mathrm{eig}}=\frac{T_{a} k}{2 \hat{B}} \operatorname{tr} A A^{\mathrm{as}}=0 .
$$

\section{APPENDIX F: FORMULATION OF THE \\ DIAGRAMMATIC APPROACH TO CALCULATING THE ENERGY FLUX}

We provide the mathematical formulation of the diagrammatic approach in Appendixes F 1 and F 2 and its application to typical networks in Appendixes F 3 and F 5.

\section{Derivation of diagrammatic expansion}

The central object in the diagrammatic approach is $G^{\tau} \equiv G^{+}(-i / \tau)$. In the noninteracting case $(k=0), G^{\tau}$ is analytically solvable. We denote $\left.G^{\tau}\right|_{k=0}=G_{0}^{\tau}$. The inverse $\left(G_{0}^{\tau}\right)^{-1}$ has a block-diagonal form,

$$
\begin{aligned}
\left(G_{0}^{\tau}\right)^{-1} & =k_{g, \tau} I+\frac{\hat{B}}{\tau} A \\
& =\sum_{i}|i\rangle\langle i| \otimes\left(k_{g, \tau} I+\frac{\hat{B}}{\tau} A_{1}\right),
\end{aligned}
$$

where $k_{g, \tau} \equiv k_{g}+(\gamma / \tau)+\left(m / \tau^{2}\right)$. Then, $G_{0}^{\tau}$ is also block diagonal, with each block the inverse of the blocks above,

$$
\begin{gathered}
G_{0}^{\tau}=\sum_{i}|i\rangle\langle i| \otimes \frac{1}{\left(k_{g, \tau}\right)^{2}+(\hat{B} / \tau)^{2}}\left(k_{g, \tau} I-\frac{\hat{B}}{\tau} A_{1}\right) \\
=\sum_{i}|i\rangle\langle i| \otimes \frac{1}{k_{0}} R_{\alpha},
\end{gathered}
$$

where $k_{0} \equiv \sqrt{\left(k_{g, \tau}\right)^{2}+(\hat{B} / \tau)^{2}}$, and $R_{\alpha}$ is the rotation matrix with angle $\alpha \equiv \arcsin \left[(\hat{B} / \tau) / k_{0}\right]$,

$$
R_{\alpha}=\left(\begin{array}{cc}
\cos \alpha & -\sin \alpha \\
\sin \alpha & \cos \alpha
\end{array}\right)
$$

We now turn on $k$. We denote the interparticle part of the force matrix $K$ as $k K_{s}$, where the factor $k$ is extracted so that the matrix $K_{s}$ is dimensionless. The $2 \times 2$ blocks of $K_{s}$ read

$$
\left\langle i\left|K_{s}\right| i\right\rangle=\sum_{i^{\prime} \neq i} e_{i i^{\prime}} e_{i i^{\prime}}^{T}, \quad\left\langle i\left|K_{s}\right| j\right\rangle=e_{i j} e_{j i}^{T}
$$

Then, $G^{\tau}$ reads

$G^{\tau}=\frac{1}{\left(G_{0}^{\tau}\right)^{-1}+k K_{s}}=\frac{1}{k_{0}}\left[\left(k_{0} G_{0}^{\tau}\right)^{-1}+\frac{k}{k_{0}} K_{s}\right]^{-1}$.

In the small- $k / k_{0}$ regime, this matrix inversion can be expanded as

$$
\begin{aligned}
G^{\tau}= & \frac{1}{k_{0}}\left[\left(k_{0} G_{0}^{\tau}\right)+\frac{k}{k_{0}}\left(k_{0} G_{0}^{\tau}\right)\left(-K_{s}\right)\left(k_{0} G_{0}^{\tau}\right)\right. \\
& \left.+\left(\frac{k}{k_{0}}\right)^{2}\left(k_{0} G_{0}^{\tau}\right)\left(-K_{s}\right)\left(k_{0} G_{0}^{\tau}\right)\left(-K_{s}\right)\left(k_{0} G_{0}^{\tau}\right)+\ldots\right] \\
= & \frac{1}{k_{0}}\left(k_{0} G_{0}^{\tau}\right) \sum_{n=0}^{\infty}\left[\frac{k}{k_{0}}\left(-K_{s}\right)\left(k_{0} G_{0}^{\tau}\right)\right]^{n}
\end{aligned}
$$

To find the convergence radius, we can write the eigendecomposition of the matrix $\left(-K_{s}\right)\left(k_{0} G_{0}^{\tau}\right)$ as $\left(-K_{s}\right)\left(k_{0} G_{0}^{\tau}\right)=$ $W \Lambda W^{-1}$, where $\Lambda$ is the diagonal matrix that contains all eigenvalues $\lambda_{i}$ 's; then, the flux becomes 


$$
\begin{aligned}
\langle J\rangle & \propto \operatorname{tr} G^{\tau} A^{\text {as }} \propto \sum_{n=0}^{\infty} \operatorname{tr}\left(k_{0} G_{0}^{\tau}\right)\left[\frac{k}{k_{0}} W \Lambda W^{-1}\right]^{n} A^{\text {as }} \\
& =\sum_{i}\left[W^{-1} A^{\text {as }}\left(k_{0} G_{0}^{\tau}\right) W\right]_{i i} \sum_{n}\left(\frac{k}{k_{0}} \lambda_{i}\right)^{n} .
\end{aligned}
$$

For terms in the series to be convergent, $k / k_{0}$ should satisfy

$$
\frac{k}{k_{0}}<\frac{1}{\max _{i}\left|\lambda_{i}\right|}
$$

The interval of convergence depends on the geometry of the whole network, as well as the condensed parameter $\alpha$. The values of the upper bound of $k / k_{0}$ for many networks range between 0.3 and 0.6 .

We note that the matrix $A^{\text {as }}$ and $K_{s}$ have common blocks, $A^{\text {as }}=-|i\rangle\left\langle j\left|\otimes e_{i j} e_{j i}^{T}+\right| j\right\rangle\langle i| \otimes e_{j i} e_{i j}^{T} \quad$ and $\quad\left\langle i\left|K_{s}\right| j\right\rangle=$ $e_{i j} e_{j i}^{T}$, so $A^{\text {as }}$ can merge with terms in the expansion of $G^{\tau}$ :

$$
\begin{aligned}
\frac{\langle J\rangle}{T_{a} / \tau} & =-\frac{k}{2}\left(\operatorname{tr} G^{\tau} A^{\mathrm{as}}\right) \\
& =-\frac{k}{2}\left(\operatorname{tr}\left\langle i\left|G^{\tau}\right| j\right\rangle e_{j i} e_{i j}^{T}-\operatorname{tr}\left\langle j\left|G^{\tau}\right| i\right\rangle e_{i j} e_{j i}^{T}\right) \\
& =\frac{k}{2}\left(\operatorname{tr}\left\langle i\left|G^{\tau}\right| j\right\rangle\left\langle j\left|-K_{s}\right| i\right\rangle-\operatorname{tr}\left\langle j\left|G^{\tau}\right| i\right\rangle\left\langle i\left|-K_{s}\right| j\right\rangle\right) .
\end{aligned}
$$

Now, we use the expansion in Eq. (F7) and insert its $(n-1)$ th-order term into the first component in Eq. (F10), which gives $k \operatorname{tr}\langle i|\left(1 / k_{0}\right)\left(k_{0} G_{0}^{\tau}\right)\left[\left(k / k_{0}\right)\left(-K_{s}\right) \times\right.$ $\left.\left(k_{0} G_{0}^{\tau}\right)\right]^{n-1}|j\rangle\left\langle j\left|-K_{s}\right| i\right\rangle$. If $n-1=0$, this term vanishes, so we only need to consider the $n-1 \geq 1$ case. Insert the $n-1$ resolution of the identity $I=\sum_{l_{a}=1}^{N}\left|l_{a}\right\rangle\left\langle l_{a}\right|$, and plug in $k_{0} G_{0}^{\tau}$ [Eq. (F3)]; we get

$$
\begin{aligned}
& \frac{k}{k_{0}} \operatorname{tr}\left\langle i\left|\left(k_{0} G_{0}^{\tau}\right)\left[\frac{k}{k_{0}}\left(-K_{s}\right)\left(k_{0} G_{0}^{\tau}\right)\right]^{n-1}\right| j\right\rangle\left\langle j\left|-K_{s}\right| i\right\rangle \\
& =\left(\frac{k}{k_{0}}\right)^{n} \sum_{l_{1}, l_{2}, \ldots, l_{n-1}} \operatorname{tr}\left\langle i\left|\left(k_{0} G_{0}^{\tau}\right)\right| l_{n-1}\right\rangle\left\langle l_{n-1}\left|\left(-K_{s}\right)\left(k_{0} G_{0}^{\tau}\right) \cdots\right| l_{1}\right\rangle\left\langle l_{1}\left|\left(-K_{s}\right)\left(k_{0} G_{0}^{\tau}\right)\right| j\right\rangle\left\langle j\left|-K_{s}\right| i\right\rangle \\
& =\left(\frac{k}{k_{0}}\right)^{n} \sum_{l_{1}, l_{2}, \ldots, l_{n-2}} \operatorname{tr} R_{\alpha}\left(-K_{s}\right)_{i l_{n-2}} R_{\alpha} \cdots\left(-K_{s}\right)_{l_{1} j} R_{\alpha}\left(-K_{s}\right)_{j i},
\end{aligned}
$$

where $\left(-K_{s}\right)_{l_{b} l_{a}} \equiv\left\langle l_{b}\left|-K_{s}\right| l_{a}\right\rangle$. We denote the path $l: i \rightarrow j \rightarrow l_{1} \rightarrow l_{2} \rightarrow \ldots \rightarrow l_{n-2} \rightarrow i$, and its corresponding term in the above summation as $S_{l}$,

$$
S_{l}=\left(\frac{k}{k_{0}}\right)^{n} \operatorname{tr} R_{\alpha}\left(-K_{s}\right)_{i l_{n-2}} R_{\alpha} \cdots\left(-K_{s}\right)_{l_{1} j} R_{\alpha}\left(-K_{s}\right)_{j i} .
$$

The second term of the flux in Eq. (F10) can be treated similarly, and it results in $S_{-l}$, where $-l$ means path $l$ in reverse order. Combining Eqs. (F12) and (F10), we get the diagrammatic expansion of the flux,

$$
\frac{\langle J\rangle}{T_{a} / \tau}=\sum_{l} J_{l}^{\mathrm{path}}=\sum_{l} \frac{1}{2}\left(S_{l}-S_{-l}\right) .
$$

\section{Path rules and properties}

The path rules can be extracted from the expression of $S_{l}$ and $J^{\text {path }}$. From the element $(-K)_{l_{b} l_{a}}$ in $S_{l}$, we see that either $l_{a}, l_{b}$ are bonded or $l_{a}=l_{b}$; otherwise $(-K)_{l_{b} l_{a}}=0$. So the path has to be a closed walk along the edges of the network. From $J_{l}^{\text {path }}$ for the flux from $i$ to $j$, we see that if the path contains equal numbers of $i \rightarrow j$ and $j \rightarrow i$, the net contribution is zero. Either $-l=l\left(\right.$ so $\left.J_{l}^{\text {path }} \propto S_{l}-S_{-l}=0\right)$ or $-l=l^{\prime}$ is another path in the summation, and $J_{l}^{\mathrm{path}}+J_{l^{\prime}}^{\mathrm{path}}=0$.

To calculate $S_{-l}$, there is a convenient way, given that $S_{l}$ is known. Based on the transformation below, $S_{-l}$ can be obtained by taking the result of $S_{l}$ and then replacing $\alpha$ by $-\alpha$ :

$$
\begin{aligned}
\frac{S_{-l}}{\left(\frac{k}{k_{0}}\right)^{n}} & =\operatorname{tr}\left(R_{\alpha}\left(-K_{s}\right)_{i j} R_{\alpha}\left(-K_{s}\right)_{j l_{1}} \cdots R_{\alpha}\left(-K_{s}\right)_{l_{n-2} i}\right)^{T} \\
& =\operatorname{tr} R_{-\alpha}\left(-K_{s}\right)_{i l_{n-2}} R_{-\alpha} \cdots\left(-K_{s}\right)_{l_{1} j} R_{-\alpha}\left(-K_{s}\right)_{j i}
\end{aligned}
$$

To interpret $S_{l}$ in a more heuristic way, we insert $I=$ $e_{i j} e_{i j}^{T}+e_{i j, \perp} e_{i j, \perp}^{T}$ into the trace in Eq. (F12), where $e_{i j, \perp}$ denotes the unit direction perpendicular to $e_{i j}$. Because $\left(-K_{s}\right)_{j i} e_{i j, \perp}=0$, the trace reduces to a matrix product

$\frac{S_{l}}{\left(\frac{k}{k_{0}}\right)^{n}}=e_{i j}^{T} R_{\alpha}\left(-K_{s}\right)_{i l_{n-2}} R_{\alpha} \cdots\left(-K_{s}\right)_{l_{1} j} R_{\alpha}\left(-K_{s}\right)_{j i} e_{i j}$

This expression can be interpreted as the following sequence of operations: Starting from a unit displacement 
of $i$ along $e_{i j}, j$ would be displaced according to the force $\left(-K_{s}\right)_{j i} e_{i j}$, after which $j$ is rotated by angle $\alpha$; then, we start from $j$ and perform similar operations for $\left(-K_{s}\right)_{l_{1} j}$ and $R_{\alpha}$; finally, the transmission goes back to $i$. We project the displacement onto $e_{i j}$, and this value is $S_{l}$ [apart from the prefactor $\left(k / k_{0}\right)^{n}$ ].

The paths can be pictorially represented by diagrams, from which the flux $J_{l}^{\text {path }}$ can be calculated easily. For instance, the first diagram in Fig. 3(a) represents the path $1 \rightarrow 2 \rightarrow 3 \rightarrow 1$. To calculate $S_{l}$, we write down $\left(-K_{s}\right)_{l_{b} l_{a}}$ for each arrow $l_{a} \rightarrow l_{b}, R_{\alpha}$ for each node $l_{a}$; we then multiply these matrices in the reverse order and calculate the trace, e.g., $S_{1 \rightarrow 2 \rightarrow 3 \rightarrow 1}=\left(k / k_{0}\right)^{3} \operatorname{tr} R_{\alpha}\left(-K_{s}\right)_{13} R_{\alpha}\left(-K_{s}\right)_{32} R_{\alpha}\left(-K_{s}\right)_{21}$. To get $S_{-l}$, we take the result of $S_{l}$ and replace $\alpha$ by $-\alpha$. Finally, $J_{l}^{\text {path }}$ can be calculated from the difference between $S_{l}$ and $S_{-l}$.

A useful property of the diagrams can be seen from Eq. (F12) and the form of $\left(-K_{s}\right)_{j i}$ in Eq. (F5). If a diagram contains no loops (e.g., $l_{a} \rightarrow l_{a}$ ) on a node, then the diagram is equal to a simplified one where we remove the node's other neighbors that do not form arrows with the node.

\section{Flux of polygon diagrams}

Here, we write down the flux formula for a polygon path without loops. It is easier to work in local coordinates, where each node has its own coordinate system. Note that now the force $F_{i j}$ in the flux definition, Eq. (4), does not necessarily equal $-F_{j i}$ because these two vectors are expressed under different coordinates or bases. However, it can be proven that the expression for energy flux is still given by Eq. (10), and diagrammatic techniques yield the same result, Eq. (11).

For each node $i$ in the path, we establish a local coordinate such that the angle from $i$ to $i-1$ is $\pi$, and the angle from $i$ to $i+1$ is $\theta_{i}$. Then, the matrix $\left(-K_{s}\right)_{i+1, i}$ reads

$$
\left(-K_{s}\right)_{i+1, i}=-e_{i+1, i} e_{i, i+1}^{T}=\left(\begin{array}{l}
1 \\
0
\end{array}\right)\left(\cos \theta_{i} \sin \theta_{i}\right)
$$

The trace in $S_{l}$ becomes

$$
\begin{aligned}
& \frac{S_{l}}{\left(\frac{k}{k_{0}}\right)^{n}}=\operatorname{tr} \prod_{i}\left(-K_{s}\right)_{i+1, i} R_{\alpha} \\
& =\operatorname{tr} \prod_{i}\left(\begin{array}{l}
1 \\
0
\end{array}\right)\left(\begin{array}{ll}
\cos \theta_{i} & \sin \theta_{i}
\end{array}\right) R_{\alpha} \\
& =\prod_{i}\left(\begin{array}{cc}
\cos \theta_{i} & \sin \theta_{i}
\end{array}\right) R_{\alpha}\left(\begin{array}{l}
1 \\
0
\end{array}\right) .
\end{aligned}
$$

So the flux for this path without loops can be written

$$
J_{\text {polygon }}^{\text {path }}=\frac{1}{2}\left(\frac{k}{k_{0}}\right)^{n}\left(\prod_{i} \cos \left(\theta_{i}-\alpha\right)-\prod_{i} \cos \left(\theta_{i}+\alpha\right)\right) .
$$

\section{Contribution from higher-order diagrams}

In some situations, the contribution of polygon diagrams vanishes, and higher-order paths involving loops become dominant. Unlike the polygon paths, paths with loops are affected by side chains.

One situation is when the polygon path itself vanishes. In Fig. 8(a), the flux of the lowest-order path, square [Fig. 8(b)], is zero, so the main contribution comes from the path with length 5 [Fig. 8(b)]. Through the loop in this path, the orientation of the side chain controls the flux direction in the main square, without changing the geometry of the main cycle [as seen in Fig. 1(b)].

Another situation is where two polygon diagrams cancel each other, which happens in honeycomblike networks away from the boundary [Fig. 9(a)]. With careful calculations, the fluxes for $n_{l} \geq 1$ are not exactly zero; rather, they appear as an exponential decay [Fig. 9(b)]. By changing the geometric angle $\theta$, the decay length varies nonmonotonically and has a cusp at $\theta=\alpha$ [Fig. 9(c)]. This decay and its relationship with $\theta$ can be explained by considering the diagrams. While the hexagon path constitutes the lowest-order diagram at the boundary, it vanishes for $n_{l} \geq 1$ due to cancellations. The first nonvanishing pair of diagrams for $n_{l}=1$ is shown in Fig. 9(d), in which the loop exploits the asymmetry between the bulk side (with a vertical bond at the blue loop) and the boundary side (with no vertical bonds at the red loop). For every increment of one layer, the length of the paths increases by 4 . So, the flux at layer $n_{l}$ is on the order of $k^{4 n_{l}+3}$, which exhibits an exponential decay $e^{-\left(n_{l}-1\right) / d_{l}}$. Through the calculation of these paths, we get the decay length $d_{l}=-1 / \log \left[4\left(k / k_{0}\right)^{4}(\sin (\theta+\alpha) \sin (\theta-\alpha))^{2}\right]$. From this

(a)
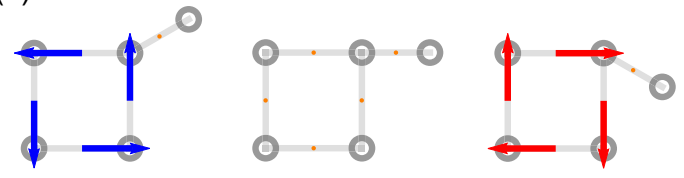

(b)
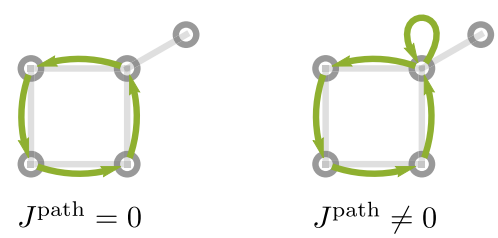

FIG. 8. Higher-order diagrams in a tailed-square network. (a) Direction of the flux can be controlled by the orientation of the side chain. (b) From the diagrammatic approach, the flux of the lowest-order diagram (square) vanishes, and the first nonvanishing diagram is affected by the side chain. 
(a)

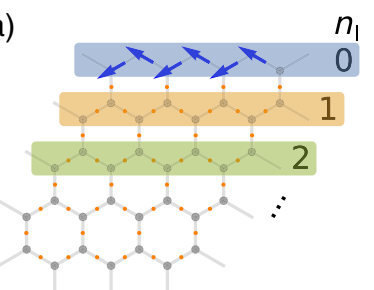

(c)

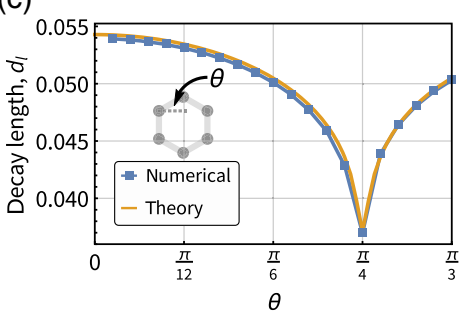

(b)

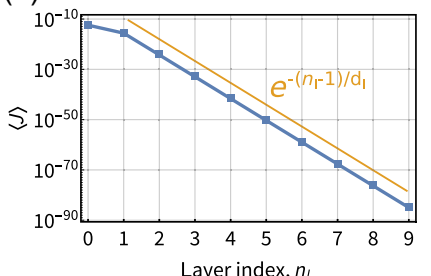

(d)

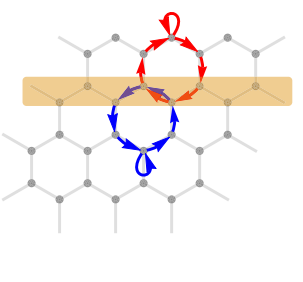

FIG. 9. Decay of fluxes away from the boundary of honeycomb networks, and explanation using the diagrammatic technique. (a) Schematic of a honeycomb network that is periodic in the $x$ direction. Layers from the boundary are indexed as $n_{l}$. (b) Semilog plot of flux $\langle J\rangle$ at layer $n_{l}$. The flux starting from layer $n_{l}=1$ shows exponential decay, with decay length $d_{l}$. Parameters used for numerical calculations are $\theta=\pi / 6$, $k / k_{0}=0.01, \alpha=\pi / 4$. (c) Decay length $d_{l}$, which changes with the network angle $\theta$ nonmonotonically, and the curve, which has a cusp at $\theta=\alpha=\pi / 4$. At small $k / k_{0}$, perturbation theory results agree with numerical calculations. (d) The first nonvanishing diagram pair for $n_{l}=1$ with length 7 . The two diagrams do not cancel because the loop in the bulk and that at the boundary have different values.

result, we see that the cusp at $\theta=\alpha$ in Fig. 9(c) is due to the term $\sin (\theta-\alpha)$. In fact, at the special point $\theta=\alpha$, paths like Fig. 9(d) vanish, and we need to consider even higherorder paths.

\section{Exact flux for 1D networks}

We show below that the flux of the 1D network is exactly zero. For simplicity, we align all sites on the $x$ axis.

We first consider cases where only nearest-neighbor connections are allowed. The $1 \mathrm{D}$ chain effectively has $C 2$ symmetry (bond lengths are irrelevant for the dynamics), so the flux should also obey $C 2$ symmetry, which means that the flux can only be zero.

Now, we consider more general cases, where bondings between non-neighboring sites are allowed. In such cases, the $1 \mathrm{D}$ system does not necessarily obey $C 2$ symmetry, and the symmetry argument above cannot be applied. However, we can use the diagrammatic approach formulated in Eqs. (11) and (F12).

For an arbitrary diagram, we look at terms in the expression $S_{ \pm l}$,

$$
\left(-K_{s}\right)_{l_{a} l_{a}}=-\sum_{l_{b} \neq l_{a}}\left(\begin{array}{c}
\lambda_{l_{a} l_{b}} \\
0
\end{array}\right)\left(\begin{array}{ll}
\lambda_{l_{a} l_{b}} & 0
\end{array}\right),
$$

$$
\left(-K_{s}\right)_{l_{a} l_{b},\left(l_{a} \neq l_{b}\right)}=-\left(\begin{array}{c}
\lambda_{l_{a} l_{b}} \\
0
\end{array}\right)\left(\begin{array}{ll}
\lambda_{l_{b} l_{a}} & 0
\end{array}\right),
$$

where $\lambda_{l_{a} l_{b}} \equiv \cos \theta_{l_{a} \rightarrow l_{b}}$ equals 1 if the bond from $l_{a}$ to $l_{b}$ lies in the $+x$ direction, and it equals -1 if the bond lies in the $-x$ direction.

We plug $\left(-K_{s}\right)_{l_{a} l_{b}}$ into the expression for $S_{l}$ and get

$$
\begin{gathered}
S_{l}=\left(\frac{-k}{k_{0}}\right)^{n}\left[\left(\begin{array}{cc}
\lambda_{i j} & 0
\end{array}\right) R_{\alpha}\left(\begin{array}{c}
\lambda_{i l_{n}} \\
0
\end{array}\right)\left(\begin{array}{cc}
\lambda_{l_{n} i} & 0
\end{array}\right)\right. \\
\left.\cdots R_{\alpha}\left(\begin{array}{c}
\lambda_{l_{3} j} \\
0
\end{array}\right)\left(\begin{array}{ll}
\lambda_{j l_{3}} & 0
\end{array}\right) R_{\alpha}\left(\begin{array}{c}
\lambda_{j i} \\
0
\end{array}\right)\right] .
\end{gathered}
$$

Terms from $\left(-K_{s}\right)_{l_{a} l_{a}}$ can be similarly considered.

We see that $S_{l}$ is a product of terms like $\left(\begin{array}{ll}\lambda_{l_{a} l_{b}} & 0\end{array}\right) R_{\alpha}\left(\begin{array}{c}\lambda_{l_{c} l_{d}} \\ 0\end{array}\right)=\lambda_{l_{a} l_{b}} \lambda_{l_{c} l_{d}} \cos \alpha$, which is an even function of $\alpha$; thus, $S_{-l}=S_{l}(-\alpha)=S_{l}(\alpha)$. The flux for path $l$ vanishes, $J_{l}^{\text {path }} \propto \sum_{l}\left(S_{l}-S_{-l}\right)=0$.

This equality holds for any diagram $l$, so the exact flux as a sum over all the diagrams vanishes. The result is exact beyond the small- $k$ regime due to analytic continuation. The brief message extracted from the derivation is that, although the particles are allowed to move in $2 \mathrm{D}$, the "statistical" effect of the $B$ field has to be projected onto and transmitted through the bonds, but if all bonds are 1D collinear, the projection does not distinguish $B$ from $-B$; thus, the effect of the $B$ field is nullified.

\section{APPENDIX G: GENERALIZATION OF THE DIAGRAMMATIC APPROACH TO A BROADER RANGE OF SYSTEMS}

The system we started with is a class of $2 \mathrm{D}$ linearized spring-mass networks, where each particle also experiences a Lorentz force, a friction, and an OU colored noise. Here, we show that the diagrammatic approach originally developed for this class of systems, can be generalized in its embedding space and in the spectrum of its noise.

\section{Generalization of 2D planar space}

The 2D space can be generalized to a $2 \mathrm{D}$ curved surface or 3D space.

For a 2D curved surface, we assume linearized dynamics, where each site moves on its local tangent plane, and the $B$ field aligns with the normal at each site. The same diagrammatic expansion as formulated in Eqs. (11) and (12) can be readily applied. The reason is that we adopted a local coordinate system (Appendix F 3), which holds both for the planar surface and for the curved surface. Curvature can affect the relation between outer angles $\theta_{i}$ and the number of sides $n$ in a polygon path. For instance, consider a polygon with all $\theta_{i}=\pi / 2$; then, Eq. (12) dictates 
$J_{\text {polygon }}^{\text {path }} \propto \sin ^{n} \alpha-\sin ^{n}(-\alpha)$. On a planar surface, such a polygon is a rectangle $(n=4), J_{\text {polygon }}^{\text {path }}=0$, whereas for a curved surface, such a polygon can be a spherical triangle $(n=3), J_{\text {polygon }}^{\text {path }} \propto \sin ^{3} \alpha \neq 0$.

For 3D space, we assume that the $B$ field remains the same as in the $2 \mathrm{D}$ planar case. The same diagrammatic approach applies, but the expressions get modified by the additional spatial dimension. We use local spherical coordinates and denote the angle with the $z$ axis as $\phi$. The noninteracting part of the response matrix, Eq. (4), is modified to

$\left(G_{0}^{\tau}\right)_{3 D}=\sum_{i}|i\rangle\langle i| \otimes \frac{1}{k_{0}}\left(\begin{array}{ccc}\cos \alpha & -\sin \alpha & 0 \\ \sin \alpha & \cos \alpha & 0 \\ 0 & 0 & \frac{1}{\cos \alpha}\end{array}\right)$.

For a polygon path, the bond vectors read

$$
\begin{gathered}
\left(e_{i+1, i}\right)_{3 D}=\left(\begin{array}{ccc}
-1 & 0 & 0
\end{array}\right)^{T}, \\
\left(e_{i, i+1}\right)_{3 D}=\left(\begin{array}{lll}
\sin \phi_{i} \cos \theta_{i} & \sin \phi_{i} \sin \theta_{i} & \cos \phi_{i}
\end{array}\right)^{T} .
\end{gathered}
$$

Plugging these modified expressions into $S_{l}$, Eq. (F12), we get the flux for 3D,

$$
\begin{aligned}
\frac{\left(J_{\text {polygon }}^{\text {path }}\right)_{3 D}}{T_{a} / \tau}= & \frac{1}{2}\left(\frac{k}{k_{0}}\right)^{n}\left[\prod_{i} \cos \left(\theta_{i}-\alpha\right) \sin \phi_{i}\right. \\
& \left.-\prod_{i} \cos \left(\theta_{i}+\alpha\right) \sin \phi_{i}\right],
\end{aligned}
$$

which effectively calculates a projection onto the $x y$ plane.

\section{Generalization of OU color noise}

The OU colored noise can be generalize to any noise with a spectrum $\left\langle\tilde{\eta}(\omega) \tilde{\eta}\left(\omega^{\prime}\right)^{T}\right\rangle=2 \gamma T_{a} h(\omega) I \delta_{\omega+\omega^{\prime}, 0} / t$. The procedure in deriving the diagrammatic expansion in Appendix $\mathrm{F} 1$ still applies, except now the noninteracting part of $G^{+}(\omega=-i / \tau)$ is replaced by $G^{+}(\omega)$ at the general $\omega$. As a result, the path rules remain unchanged, but the mathematical expressions for the diagrams are modified.

We first define an auxiliary function $J^{\text {aux }}(\omega)$,

$$
J^{\operatorname{aux}}(\omega)=-k \operatorname{tr} G^{+}(\omega) A^{\text {as }} .
$$

Following Appendix F1, we apply a diagrammatic expansion to $J^{\text {aux }}(\omega)$ and get

$$
\begin{gathered}
J^{\text {aux }}(\omega)=\sum_{l} J_{l}^{\text {aux,path }}(\omega)=\sum_{l}\left(S_{\omega, l}-S_{\omega,-l}\right), \\
S_{\omega, l}=\left(\frac{k}{k_{0, \omega}}\right)^{n}\left\{\operatorname{tr} R_{\alpha_{\omega}}\left(-K_{s}\right)_{i l_{n-2}} R_{\alpha_{\omega}}\right. \\
\left.\cdots\left(-K_{s}\right)_{l_{1} j} R_{\alpha_{\omega}}\left(-K_{s}\right)_{j i}\right\}
\end{gathered}
$$

where the symbols are defined as

$$
\begin{gathered}
k_{0, \omega}=\sqrt{\left(k_{g}+i \omega \gamma-m \omega^{2}\right)^{2}-(\omega \hat{B})^{2}}, \\
R_{\alpha_{\omega}}=\left(\begin{array}{cc}
\cos \alpha_{\omega} & -\sin \alpha_{\omega} \\
\sin \alpha_{\omega} & \cos \alpha_{\omega}
\end{array}\right), \\
\cos \alpha_{\omega}=\frac{1}{k_{0, \omega}}\left(k_{g}+i \omega \gamma-m \omega^{2}\right), \\
\sin \alpha_{\omega}=\frac{1}{k_{0, \omega}} i \omega \hat{B} .
\end{gathered}
$$

Note that the condensed parameters $k_{0, \omega}, \alpha_{\omega}$ are complex, as opposed to the real $k_{0}, \alpha$ for the case with OU noise [Eq. (13)].

For polygon paths with outer angles $\theta_{i}$ 's, $S_{\omega, \pm l}$ simplifies to

$$
S_{\omega, \pm l}=\left(\frac{k}{k_{0, \omega}}\right)^{n} \prod_{i} \cos \left(\theta_{i} \mp \alpha_{\omega}\right),
$$

which is formally similar to $S_{l}$ for the OU noise case [Eq. (12)].

Based on the spectral expression, Eq. (7), the flux $\langle J\rangle$ can be calculated in two ways. One way is to write $J^{F T}(\omega)$ in terms of $J^{\text {aux }}(\omega)$,

$$
J^{F T}(\omega)=\frac{T_{a}}{4 \pi}\left(J^{\text {aux }}(\omega)+J^{\text {aux }}(-\omega)\right),
$$

and then express $\langle J\rangle$ as an integral, $\langle J\rangle=$ $\int d \omega h(\omega) J^{F T}(\omega)$.

Another way is to first integrate Eq. (7) using the residue theorem and then express $\langle J\rangle$ in terms of $J^{\text {aux }}(\omega)$ evaluated at the poles,

$$
\langle J\rangle=-T_{a} i \sum_{i} \operatorname{Res}_{\omega=\omega_{i}}\left[h(\omega) J^{\mathrm{aux}}(\omega)\right] .
$$

\section{APPENDIX H: SIMULATION PROTOCOL FOR ACTIVE GYROSCOPIC SYSTEMS}

The simulation is performed using LAMMPS [53] with the MOLTEMPLATE toolkit [54] and custom code. We use a Trotter splitting method $[55,56]$ to simulate the underdamped Langevin dynamics. The integrator combines the integrator for colored noise [37] and that for Lorentz force [57]. We did not simulate the commonly used overdamped Langevin dynamics because some intricacy arises when the system also experiences a Lorentz force [58]. Below, we first define each step in the integrator and then present the combined result.

The velocity-Verlet step $U_{v v}$ is the integrator when both the Lorentz force and the colored noise are absent. It is defined as 


$$
\begin{gathered}
U_{v v}(\Delta t): v \leftarrow v+F(x) \Delta t /(2 m) \\
x \leftarrow x+v \Delta t \\
v \leftarrow v+F(x) \Delta t /(2 m),
\end{gathered}
$$

where $F(x)$ is the conservative force, including on-site and interparticle potentials.

Writing the Lorentz force part as

$$
\dot{v}=-\left(\begin{array}{cc}
0 & \hat{B} / m \\
-\hat{B} / m & 0
\end{array}\right)\left(\begin{array}{l}
v_{x} \\
v_{y}
\end{array}\right) \equiv-a_{p} v,
$$

its integrator $U_{L}$ is a rotation of the velocity

$$
U_{L}(\Delta t): v \leftarrow e^{-\Delta t a_{p}} v .
$$

Writing the colored noise part as

$$
\begin{gathered}
\frac{d}{d t}\left(\begin{array}{c}
v \\
\eta
\end{array}\right)=-A_{p}\left(\begin{array}{l}
v \\
\eta
\end{array}\right)+B_{p}\left(\begin{array}{c}
\xi_{w} \\
\xi_{a}
\end{array}\right), \\
A_{p}=\left(\begin{array}{cc}
\frac{\gamma}{m} & -\frac{1}{m} \\
0 & \frac{1}{\tau}
\end{array}\right), \quad B_{p}=\left(\begin{array}{cc}
0 & 0 \\
0 & \frac{\sqrt{2 \gamma T_{a}}}{\tau}
\end{array}\right),
\end{gathered}
$$

its integrator $U_{\text {OUP }}$ reads

$$
U_{\mathrm{OUP}}(\Delta t):\left(\begin{array}{c}
v \\
\eta
\end{array}\right) \leftarrow T(\Delta t)\left(\begin{array}{c}
v \\
\eta
\end{array}\right)+S(\Delta t)\left(\begin{array}{c}
0 \\
N_{a}
\end{array}\right),
$$

where $N_{a}$ is the standard Gaussian random variable, and

$$
\begin{gathered}
T(\Delta t)=e^{-\Delta t A_{p}}, \\
S(\Delta t) S(\Delta t)^{T}=C_{p}-T(\Delta t) C_{p} T(\Delta t)^{T} .
\end{gathered}
$$

Here, $C_{p}$ is the solution of $A_{p} C_{p}+C_{p} A_{p}^{T}=B_{p} B_{p}^{T}$, and $S(\Delta t)$ can be solved as an upper-triangle matrix.

Combining these steps together, the integrator for one time step $\Delta t$ reads

$$
\begin{aligned}
U(\Delta t)= & U_{\text {OUP }}\left(\frac{\Delta t}{2}\right) U_{L}\left(\frac{\Delta t}{2}\right) U_{v v}(\Delta t) U_{L} \\
& \times\left(\frac{\Delta t}{2}\right) U_{\text {OUP }}\left(\frac{\Delta t}{2}\right),
\end{aligned}
$$

where the order of operations is from right to left.

\section{APPENDIX I: RELATIONSHIP BETWEEN SWIMMER'S SPEED AND ENERGY FLUX}

To understand the proportionality between $V_{s}$ and $\langle J\rangle$, we turn to the diagrammatic technique. Different from previous cases, this path sum can be computed exactly, so the result holds beyond the small- $k$ regime.

First, we rewrite $V_{s}$ as follows:

$$
\frac{V_{s}}{7 a / 24 L^{2}}=\left\langle J_{12}^{s}\right\rangle+\left\langle J_{23}^{s}\right\rangle+\left\langle J_{31}^{s}\right\rangle
$$

where we have defined $\left\langle J_{i j}^{s}\right\rangle \equiv\left\langle\left(x_{i}-x_{j}\right)\left(v_{i}+v_{j}\right)\right\rangle$. Note that $\left\langle J_{i j}^{s}\right\rangle$ is proportional to the energy flux via $\left\langle J_{i j}\right\rangle=$ $k_{i j}\left\langle J_{i j}^{s}\right\rangle / 2$, where $k_{12}=k_{23}=k$ and $k_{31}=0$ (because $\left\langle J_{31}\right\rangle=0$, there is no energy flux from 3 to 1$)$. We see that both $\left\langle J_{12}^{s}\right\rangle$ and $\left\langle J_{23}^{s}\right\rangle$ are proportional to the flux $\langle J\rangle$ apart from a factor $k$, so the remaining task is to find the relationship between $\left\langle J_{31}^{s}\right\rangle$ and $\langle J\rangle$ or $\left\langle J_{12}^{s}\right\rangle$.

We use a diagrammatic technique with the modification that the paths should contain only one $3 \rightarrow 1$ segment. This modification is a consequence of the fact that particles 3 and 1 are not bonded. We now illustrate the correspondence between the diagrams for $\left\langle J_{31}^{s}\right\rangle$ and for $\left\langle J_{12}^{s}\right\rangle$. For each path $l$ for $\left\langle J_{31}^{s}\right\rangle$, we can construct $n$ paths for $\left\langle J_{12}^{s}\right\rangle$ by reversing $l$ and then replacing $1 \rightarrow 3$ by $1 \rightarrow 2(\rightarrow 2)^{n} \rightarrow 3$, where $n=0,1, \ldots$. An example construction of paths is shown in Fig. 10. For $\left\langle J_{12}^{s}\right\rangle$, all of its paths can be constructed in this way. As a result, there is a 1-to- $n$ correspondence between the paths for $\left\langle J_{31}^{s}\right\rangle$ and for $\left\langle J_{12}^{s}\right\rangle$, which leads to the relationship

$$
\left\langle J_{12}^{s}\right\rangle=\frac{k}{k_{0}} \sum_{n=0}^{\infty}\left(-2 \frac{k}{k_{0}}\right)^{n}\left(-\left\langle J_{31}^{s}\right\rangle\right)
$$

$$
=\frac{k / k_{0}}{1-\left(-2 k / k_{0}\right)}\left(-\left\langle J_{31}^{s}\right\rangle\right),
$$

where $k_{0}=k_{g}+m / \tau^{2}(\hat{B}, \gamma=0$ for the passive part), and the factor $-2\left(k / k_{0}\right)$ comes from the loop $2 \rightarrow 2$. Plugging Eq. (I3) into the expression of $V_{s}$ in Eq. (I1), we obtain the proportionality

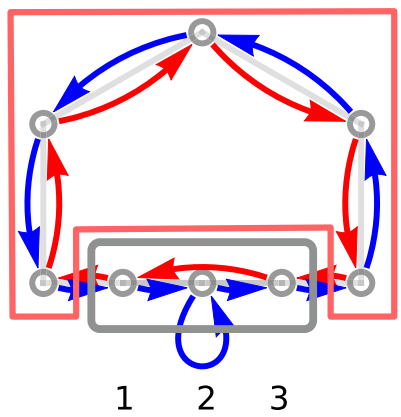

FIG. 10. An example of the $\left\langle J_{31}^{s}\right\rangle$ diagram (red) and the $\left\langle J_{12}^{s}\right\rangle$ diagram (blue). Passive particles are boxed in gray, and the active ones are boxed in red. 


$$
\frac{V_{s}}{7 a / 24 L^{2}}=-\frac{k_{0}}{k} \frac{\langle J\rangle}{k / 2},
$$

which is Eq. (15) in the main text. Since we have considered all of the diagrams, this result can be analytically continued to arbitrarily large $k$.

From this diagrammatic technique, we also see that the proportionality constant is independent of the geometry of the active part of the network because the paths through the active part for $\left\langle J_{31}^{s}\right\rangle$ and for $\left\langle J_{12}^{s}\right\rangle$ are identical.

[1] U. Seifert, Stochastic Thermodynamics, Fluctuation Theorems and Molecular Machines, Rep. Prog. Phys. 75, 126001 (2012).

[2] A. Mogilner and G. Oster, Cell Motility Driven by Actin Polymerization, Biophys. J. 71, 3030 (1996).

[3] F. Jülicher, A. Ajdari, and J. Prost, Modeling Molecular Motors, Rev. Mod. Phys. 69, 1269 (1997).

[4] P. Reimann, Brownian Motors: Noisy Transport Far from Equilibrium, Phys. Rep. 361, 57 (2002).

[5] C. J. O. Reichhardt and C. Reichhardt, Ratchet Effects in Active Matter Systems, Annu. Rev. Condens. Matter Phys. 8, 51 (2017).

[6] R. P. Feynman, R. B. Leighton, and M. Sands, The Feynman Lectures on Physics: The New Millennium Edition: Mainly Mechanics, Radiation, and Heat (Basic Books, New York, NY, USA, 2015).

[7] P. Hänggi and F. Marchesoni, Artificial Brownian Motors: Controlling Transport on the Nanoscale, Rev. Mod. Phys. 81, 387 (2009).

[8] A. Coskun, M. Banaszak, R. D. Astumian, J. F. Stoddart, and B. A. Grzybowski, Great Expectations: Can Artificial Molecular Machines Deliver on Their Promise?, Chem. Soc. Rev. 41, 19 (2012).

[9] I. A. Martínez, É. Roldán, L. Dinis, and R. A. Rica, Colloidal Heat Engines: A Review, Soft Matter 13, 22 (2017).

[10] W. Hwang and M. Karplus, Structural Basis for Power Stroke vs. Brownian Ratchet Mechanisms of Motor Proteins, Proc. Natl. Acad. Sci. U.S.A. 116, 19777 (2019).

[11] M. O. Magnasco, Forced Thermal Ratchets, Phys. Rev. Lett. 71, 1477 (1993).

[12] J. Cao, Event-Averaged Measurements of Single-Molecule Kinetics, Chem. Phys. Lett. 327, 38 (2000).

[13] S. Flach, Y. Zolotaryuk, A. E. Miroshnichenko, and M. V. Fistul, Broken Symmetries and Directed Collective Energy Transport in Spatially Extended Systems, Phys. Rev. Lett. 88, 184101 (2002).

[14] S. Das, O. Narayan, and S. Ramaswamy, Ratchet for Energy Transport between Identical Reservoirs, Phys. Rev. E 66, 050103(R) (2002).

[15] N. Li, P. Hänggi, and B. Li, Ratcheting Heat Flux Against a Thermal Bias, Europhys. Lett. 84, 40009 (2008).

[16] J. Ren and B. Li, Emergence and Control of Heat Current from Strict Zero Thermal Bias, Phys. Rev. E 81, 021111 (2010).
[17] J. Ren, S. Liu, and B. Li, Geometric Heat Flux for Classical Thermal Transport in Interacting Open Systems, Phys. Rev. Lett. 108, 210603 (2012).

[18] N. Li, J. Ren, L. Wang, G. Zhang, P. Hänggi, and B. Li, Colloquium: Phononics: Manipulating Heat Flow with Electronic Analogs and Beyond, Rev. Mod. Phys. 84, 1045 (2012).

[19] B. Sabass, Fluctuating, Lorentz-Force-Like Coupling of Langevin Equations and Heat Flux Rectification, Phys. Rev. E 96, 022109 (2017).

[20] K. Kanazawa, T. Sagawa, and H. Hayakawa, Heat Conduction Induced by Non-Gaussian Athermal Fluctuations, Phys. Rev. E 87, 052124 (2013).

[21] M. M. Cândido, W. A. M. Morgado, and S. M. D. Queirós, Macroscopic Violation of the Law of Heat Conduction, Phys. Rev. E 96, 032143 (2017).

[22] E. H. Colombo, L. A. C. A. Defaveri, and C. Anteneodo, Heat Flux Direction Controlled by Power-Law Oscillators under Non-Gaussian Fluctuations, Phys. Rev. E 100, 032118 (2019).

[23] L. Zhu and S. Fan, Persistent Directional Current at Equilibrium in Nonreciprocal Many-Body Near Field Electromagnetic Heat Transfer, Phys. Rev. Lett. 117, 134303 (2016).

[24] L. Zhu, Yu. Guo, and S. Fan, Theory of Many-Body Radiative Heat Transfer without the Constraint of Reciprocity, Phys. Rev. B 97, 094302 (2018).

[25] Y. Dubi and M. Di Ventra, Colloquium: Heat Flow and Thermoelectricity in Atomic and Molecular Junctions, Rev. Mod. Phys. 83, 131 (2011).

[26] M. C. Marchetti, J. F. Joanny, S. Ramaswamy, T. B. Liverpool, J. Prost, M. Rao, and R. A. Simha, Hydrodynamics of Soft Active Matter, Rev. Mod. Phys. 85, 1143 (2013).

[27] N. Koumakis, A. Lepore, C. Maggi, and R. Di Leonardo, Targeted Delivery of Colloids by Swimming Bacteria, Nat. Commun. 4, 2588 (2013).

[28] F. G. Woodhouse, H. Ronellenfitsch, and . Dunkel, Autonomous Actuation of Zero Modes in Mechanical Networks Far from Equilibrium, Phys. Rev. Lett. 121, 178001 (2018).

[29] L. M. Nash, D. Kleckner, A. Read, V. Vitelli, A. M. Turner, and W. T. M. Irvine, Topological Mechanics of Gyroscopic Metamaterials, Proc. Natl. Acad. Sci. U.S.A. 112, 14495 (2015).

[30] R. Süsstrunk and S. D. Huber, Classification of Topological Phonons in Linear Mechanical Metamaterials, Proc. Natl. Acad. Sci. U.S.A. 113, E4767 (2016).

[31] C. H. Lee, G. Li, G. Jin, Y. Liu, and X. Zhang, Topological Dynamics of Gyroscopic and Floquet Lattices from Newton's Laws, Phys. Rev. B 97, 085110 (2018).

[32] N. P. Mitchell, L. M. Nash, D. Hexner, A. M. Turner, and W. T. M. Irvine, Amorphous Topological Insulators Constructed from Random Point Sets, Nat. Phys. 14, 380 (2018).

[33] P. Hanggi and P. Jung, Colored Noise in Dynamical Systems, in Advances in Chemical Physics (John Wiley \& Sons, New York, 1994), pp. 239-326.

[34] S. Lepri, R. Livi, and A. Politi, Thermal Conduction in Classical Low-Dimensional Lattices, Phys. Rep. 377, 1 (2003). 
[35] K. Sekimoto, Langevin Equation and Thermodynamics, Prog. Theor. Phys. Suppl. 130, 17 (1998).

[36] C. Gardiner, The Ito Calculus and Stochastic Differential Equations, in Stochastic Methods, 4th ed. (Springer-Verlag, Berlin, Heidelberg, 2009), Chap. 4, p. 107.

[37] M. Ceriotti, G. Bussi, and M. Parrinello, Colored-Noise Thermostats à la Carte, J. Chem. Theory Comput. 6, 1170 (2010).

[38] A. Kundu, S. Sabhapandit, and A. Dhar, Large Deviations of Heat Flow in Harmonic Chains, J. Stat. Mech. (2011) P03007.

[39] T. Harada and S.-i. Sasa, Equality Connecting Energy Dissipation with a Violation of the Fluctuation-Response Relation, Phys. Rev. Lett. 95, 130602 (2005).

[40] P. Pradhan and U. Seifert, Nonexistence of Classical Diamagnetism and Nonequilibrium Fluctuation Theorems for Charged Particles on a Curved Surface, Europhys. Lett. 89, 37001 (2010).

[41] G. I. Taylor, Analysis of the Swimming of Microscopic Organisms, Proc. R. Soc. A 209, 447 (1951).

[42] E. M. Purcell, Life at Low Reynolds Number, Am. J. Phys. 45, 3 (1977).

[43] R. Golestanian and A. Ajdari, Analytic Results for the Three-Sphere Swimmer at Low Reynolds Number, Phys. Rev. E 77, 036308 (2008).

[44] R. Golestanian, Three-Sphere Low-Reynolds-Number Swimmer with a Cargo Container, Eur. Phys. J. E 25, 1 (2008).

[45] R. Golestanian and A. Ajdari, Stochastic Low Reynolds Number Swimmers, J. Phys. Condens. Matter 21, 204104 (2009).

[46] M. Leoni, J. Kotar, B. Bassetti, P. Cicuta, and M. C. Lagomarsino, A Basic Swimmer at Low Reynolds Number, Soft Matter 5, 472 (2009).
[47] Mathematica, Version 11.3, Wolfram Research, Inc. (2018).

[48] H. K. Lee, S. Lahiri, and H. Park, Nonequilibrium Steady States in Langevin Thermal Systems, Phys. Rev. E 96, 022134 (2017).

[49] H.-M. Chun and J. D. Noh, Microscopic Theory for the Time Irreversibility and the Entropy Production, J. Stat. Mech. (2018) 023208.

[50] S. Shankar and M. C. Marchetti, Hidden Entropy Production and Work Fluctuations in an Ideal Active Gas, Phys. Rev. E 98, 020604(R) (2018).

[51] L. Caprini, U. M. B. Marconi, A. Puglisi, and A. Vulpiani, The Entropy Production of Ornstein-Uhlenbeck Active Particles: A Path Integral Method for Correlations, J. Stat. Mech. (2019) 053203.

[52] L. Onsager and S. Machlup, Fluctuations and Irreversible Processes, Phys. Rev. 91, 1505 (1953).

[53] S. Plimpton, Fast Parallel Algorithms for Short-Range Molecular Dynamics, J. Comput. Phys. 117, 1 (1995).

[54] A. I. Jewett, Z. Zhuang, and J.-E. Shea, Moltemplate a Coarse-Grained Model Assembly Tool, Biophys. J. 104, 169a (2013).

[55] M. Tuckerman, B. J. Berne, and G. J. Martyna, Reversible Multiple Time Scale Molecular Dynamics, J. Chem. Phys. 97, 1990 (1992).

[56] G. Bussi and M. Parrinello, Accurate Sampling Using Langevin Dynamics, Phys. Rev. E 75, 056707 (2007).

[57] S. A. Chin, Symplectic and Energy-Conserving Algorithms for Solving Magnetic Field Trajectories, Phys. Rev. E 77, 066401 (2008).

[58] H.-M. Chun, X. Durang, and J. D. Noh, Emergence of Nonwhite Noise in Langevin Dynamics with Magnetic Lorentz, Force, Phys. Rev. E 97, 032117 (2018). 